\title{
DSMC simulation of the vertical structure of planetary rings
}

\author{
A. Frezzotti ${ }^{\star}$ \\ Dipartimento di Matematica del Politecnico di Milano Piazza Leonardo da Vinci 32 - 20133 Milano, Italy \\ Received 3 April 2001 / Accepted 4 October 2001 \\ Abstract. A statistical Monte Carlo method (DSMC) is developed to simulate granular flows occurring in dense \\ planetary rings. The accuracy of the method is assessed through a comparison with existing molecular dynamics \\ simulations of the vertical structure of planetary rings with and without self-gravity effects.
}

Key words. planets: rings

\section{Introduction}

This paper revisits the well-known problem of the determination of the vertical structure of a planetary ring. The problem is approached from the point of view of the kinetic theory of granular media and the study is based on a statistical Monte Carlo method. Unlike deterministic molecular dynamics (MD) simulations, the Direct Simulation Monte Carlo (DSMC) technique (Bird 1994) provides stochastic approximations to solutions of kinetic equations.

Initially designed to solve the Boltzmann equation for rarefied gas dynamics applications (Cercignani 2000), DSMC has been extended to dense gases (Frezzotti 1997) and to granular fluids both in the dilute (Brey et al. 1996) and in dense flow regimes (Frezzotti 1999; 2000). In the latter case it is assumed that the flow is governed by an Enskog-like kinetic equation (Enskog 1922; Goldshtein \& Shapiro 1995) which determines the evolution of the oneparticle distribution function. As is well known, Enskog's theory of dense fluids is not exact because of the approximate treatment of pair correlations. However, in spite of its phenomenological character, it has been widely applied not only to study granular flows in general (Goldshtein \& Shapiro 1995), but also granular flows in planetary rings (Araki \& Tremaine 1986; Araki 1991).

A common feature of previous kinetic theory investigations is the search for approximate solutions based either on hydrodynamic expansions (Schmidt et al. 1999) or moment method (Araki \& Tremaine 1986; Araki 1991; Simon \& Jenkins 1994), in which some reasonable Ansatz is postulated for a distribution function depending on a small number of parameters to be determined from suitably selected balance equations.

No particular ad hoc assumption on the structure of the distribution function is required by DSMC which gives the opportunity to obtain numerical but accurate

\footnotetext{
* e-mail: aldfre@mate.polini.it
}

solutions of kinetic equations. In this way, the quality of kinetic theory predictions can be clearly assessed.

Accordingly, this study aims mainly at the assessment of Enskog theory description of dense granular media through a comparison with existing MD simulations of the vertical ring structure whose kinetic theory formulation is computationally simple, being amenable to a genuine onedimensional treatment. However, this work is also meant as a first step toward more complex flows. Actually, properly designed DSMC schemes execute faster than eventdriven molecular dynamics simulations with the same particle number. Therefore, the DSMC technique might be suitable to simulate granular flows in large ring regions as required by the investigation of oscillatory instabilities which have been proposed as a possible mechanism for the formation of the fine scale structure in Saturn's B-ring (Borderies et al. 1985; Papaloizou \& Lin 1988; Mosqueira 1996; Schmit \& Tscharnuter 1995, 1999; Spahn et al. 2000).

The presentation is organized as follows: Sect. 2 is devoted to the general formulation of the problem, Sect. 3 briefly describes the numerical method. The numerical results about the vertical ring structure, with and without self-gravity effects, are presented in Sect. 4 . In Sect. 5 conclusions are drawn and a purely illustrative example of a two dimensional simulation is given which exhibits density oscillations in the ring plane.

\section{Formulation of the problem}

Following previous investigations of the local structure of planetary rings (Wisdom \& Tremaine 1988; Salo 1991a, 1991b, 1995), the motion of ring particles is studied in a region much smaller than the ring itself. This limitation is dictated by the necessity of resolving scales of the order of the grains' size for an accurate computation of collisional momentum and energy transfer in the flow field. It is assumed that the granular fluid occupying the region is composed of identical spherical particles of diameter $a$ 
and mass $m$. It is convenient to study the fluid motion in a Cartesian frame whose origin $O$ moves around the planet along a Keplerian circular orbit of radius $R$. The $x$ axis of the reference frame points radially outward, the $y$ axis is orthogonal to the $x$ axis in the equatorial plane and points in the direction of the orbital motion, whereas the $z$ axis is simply orthogonal to the equatorial plane. The physical state of each sphere is characterized by the displacement vector $\boldsymbol{x}$ of its center with respect to $O$, the relative velocity $\boldsymbol{\xi}$ of its center and by its relative angular velocity $\boldsymbol{\omega}$. The constructed reference frame is not inertial, since it rotates with angular velocity $\Omega \hat{\boldsymbol{z}}$ and its origin $O$ moves with acceleration $\boldsymbol{A}(O)=-\Omega^{2} R \hat{\boldsymbol{x}}$, being $\hat{\boldsymbol{x}}$ and $\hat{\boldsymbol{z}}$ unit vectors parallel to the $x$ axis and $z$ axis, respectively. The Keplerian frequency $\Omega$ is computed as $\Omega^{2}=G M_{\mathrm{p}} / R^{3}$, being $G$ the gravitational constant and $M_{\mathrm{p}}$ the mass of the planet.

\subsection{Collisionless particles motion}

In the time between two collisions the particles are subject to the following force field:

$\boldsymbol{F}^{\mathrm{tot}}(\boldsymbol{x}, \boldsymbol{\xi} \mid t)=\boldsymbol{F}^{\mathrm{ext}}(\boldsymbol{x}, \boldsymbol{\xi} \mid t)+\boldsymbol{F}^{\mathrm{sg}}(\boldsymbol{x} \mid t)$

where $\boldsymbol{F}^{\text {ext }}$, defined as

$$
\begin{aligned}
\boldsymbol{F}^{\mathrm{ext}}(\boldsymbol{x}, \boldsymbol{\xi} \mid t)= & -G \frac{M_{\mathrm{p} m}}{\|\boldsymbol{r}\|^{3}} \boldsymbol{r}-m[\boldsymbol{A}(O) \\
& +\boldsymbol{\Omega} \wedge(\boldsymbol{\Omega} \wedge \boldsymbol{x})+2 \boldsymbol{\Omega} \wedge \boldsymbol{\xi}] \\
\boldsymbol{r}= & R \hat{\boldsymbol{x}}+\boldsymbol{x}
\end{aligned}
$$

represents the sum of the gravitational force of the planet and the inertial forces, given by the second term on the right hand side of Eq. (2). $\boldsymbol{F}^{\mathrm{sg}}$ is the self-gravity force generated by the ring particles themselves. An evolution equation for the particles angular velocities is also needed, since in the rotating reference frame the angular velocity of a particle is not constant:

$$
\frac{\mathrm{d} \boldsymbol{\omega}}{\mathrm{d} t}=-\boldsymbol{\Omega} \wedge \boldsymbol{\omega}
$$

The mathematical treatment can be greatly simplified by taking into account that, the ring region dimensions being small in comparison to $R$, it is possible to linearize the gravitational force term and obtain the following expressions for the accelerations of the particles centers (Hill 1878)

$$
\begin{aligned}
& \ddot{x}=3 \Omega^{2} x+2 \Omega \xi_{y}+\frac{F_{x}^{\mathrm{sg}}}{m}, \\
& \ddot{y}=-2 \Omega \xi_{x}+\frac{F_{y}^{\mathrm{sg}}}{m} \\
& \ddot{z}=-\Omega^{2} z+\frac{F_{z}^{\mathrm{sg}}}{m} .
\end{aligned}
$$

\subsection{Simplified collision model}

As is usual in microscopic theories of granular flows, a phenomenological model (Lun \& Savage 1987) is used to describe the collision of two fluid particles, an otherwise complex problem in solid mechanics. It is assumed that the relative velocity at the contact point of the colliding spheres before the collision, $\boldsymbol{W}$, determines the post collisional value of the same quantity, $\boldsymbol{W}^{*}$, through the following relationship:

$\boldsymbol{W}^{*}=-e(\boldsymbol{W} \circ \boldsymbol{k}) \boldsymbol{k}-\beta \boldsymbol{W}_{t}, \quad 0 \leq e \leq 1,-1 \leq \beta \leq 1 .(5)$

In Eq. (5) $\boldsymbol{W}$ is defined as:

$\boldsymbol{W}=\boldsymbol{\xi}_{1}-\boldsymbol{\xi}-\frac{a}{2}\left(\boldsymbol{\omega}+\boldsymbol{\omega}_{1}\right)$.

The unit vector $\boldsymbol{k}=\left(\boldsymbol{x}_{1}-\boldsymbol{x}\right) /\left\|\boldsymbol{x}_{1}-\boldsymbol{x}\right\|$ has the direction of the line joining the centers of the spheres at the time of impact, whereas $\boldsymbol{W}_{t}$ is the component of $\boldsymbol{W}$ in the plane normal to $\boldsymbol{k}$. As shown by Eq. (5), $\boldsymbol{W}^{*}$ is obtained by a linear combination of the components of $\boldsymbol{W}$ along $\boldsymbol{k}$ and normal to $\boldsymbol{k}$. Experiments and theory (Brilliantov \& Pöschel 2001 and references therein) show that the normal restitution coefficient $e$ and the tangential restitution coefficient $\beta$, which appear in the linear combination, do depend on the magnitude of the relative velocity. The results described in this paper have been obtained under the assumption of velocity-independent restitution coefficients. In fact, this work aims mainly at comparing the predictions of kinetic theory with MD simulations of the same physical system in the simplest case.

The equations of conservation of total momentum and individual angular momentum with respect to the impact point, supplemented with Eq. (5), yield:

$\boldsymbol{\xi}^{*}=\boldsymbol{\xi}+\eta_{1}(\boldsymbol{W} \circ \boldsymbol{k}) \boldsymbol{k}+\eta_{2} \boldsymbol{W}_{t}$

$\boldsymbol{\omega}^{*}=\boldsymbol{\omega}-\frac{m a}{2 I} \eta_{2}(\boldsymbol{k} \wedge \boldsymbol{W})$

$\boldsymbol{\xi}_{1}^{*}=\boldsymbol{\xi}_{1}-\eta_{1}(\boldsymbol{W} \circ \boldsymbol{k}) \boldsymbol{k}-\eta_{2} \boldsymbol{W}_{t}$

$\boldsymbol{\omega}_{1}^{*}=\boldsymbol{\omega}_{1}-\frac{m a}{2 I} \eta_{2}(\boldsymbol{k} \wedge \boldsymbol{W})$

where $\eta_{1}=(1+e) / 2, \eta_{2}=\frac{\kappa(1+\beta)}{2(1+\kappa)}, \kappa=4 I / m a^{2}$ and $I$ the moment of inertia of a particle.

The collision model sketched above describes inelastic collisions. Actually, it can be easily shown that the following amount of kinetic energy is lost in each impact:

$\Delta E=-m\left[\frac{1-e^{2}}{4}(\boldsymbol{W} \circ \boldsymbol{k})^{2}+\frac{\kappa\left(1-\beta^{2}\right)}{4(1+\kappa)} \boldsymbol{W}_{t}^{2}\right]$.

The mechanical energy lost is dissipated into the grains whose internal energy is increased. It is also worth noting that kinetic energy is conserved in a collision only in the following two cases:

1. $e=1, \beta=1, \boldsymbol{W}^{*}=-\boldsymbol{W}$, elastic and perfectly rough spheres;

2. $e=1, \beta=-1, \boldsymbol{W}_{t}^{*}=\boldsymbol{W}_{t}$, elastic smooth spheres: rotational dynamics is decoupled from translational.

\subsection{The governing equation}

After specifying particle dynamics and interactions a kinetic equation can be readily obtained from the pseudoLiouville equation for an assembly of inelastically colliding 
spherical bodies (Resibois \& De Leener 1977; van Noije et al. 1998):

$$
\begin{aligned}
& \frac{\partial f_{1}}{\partial t}+\boldsymbol{\xi} \circ \nabla_{\boldsymbol{x}} f_{1}+\nabla_{\boldsymbol{\gamma}} \circ\left(\dot{\gamma}^{\mathrm{ext}} f_{1}\right)= \\
& -\nabla_{\boldsymbol{\xi}} \circ\left[\int_{x_{r}>a} \boldsymbol{k} \frac{\mathrm{d} \phi}{\mathrm{d} x_{r}} f_{2}\left(\boldsymbol{x}, \boldsymbol{\gamma}, \boldsymbol{x}_{1}, \boldsymbol{\gamma}_{1} \mid t\right) \mathrm{d} \boldsymbol{x}_{1} \mathrm{~d} \boldsymbol{\gamma}_{1}\right] \\
& +a^{2} \int\left[f_{2}\left(\boldsymbol{x}, \boldsymbol{\gamma}^{\prime}, \boldsymbol{x}+a \boldsymbol{k}, \boldsymbol{\gamma}_{1}^{\prime}\right) \Lambda-f_{2}\left(\boldsymbol{x}, \boldsymbol{\gamma}, \boldsymbol{x}-a \boldsymbol{k}, \boldsymbol{\gamma}_{1}\right)\right] \\
& \times\left(\boldsymbol{\xi}_{r} \circ \boldsymbol{k}\right)^{+} \mathrm{d} \boldsymbol{\gamma}_{1} \mathrm{~d}^{2} \boldsymbol{k} .
\end{aligned}
$$

In the equation written above $f_{1}(\boldsymbol{x}, \boldsymbol{\gamma} \mid t)$ is the oneparticle distribution function, $\gamma$ being a short-hand notation for the six-dimensional velocity vector $(\boldsymbol{\xi}, \boldsymbol{\omega})$, $f_{2}\left(\boldsymbol{x}, \boldsymbol{\gamma}, \boldsymbol{x}_{1}, \gamma_{1} \mid t\right)$ is the pair distribution function whereas $\dot{\boldsymbol{\gamma}}^{\text {ext }}=\left(\boldsymbol{F}^{\text {ext }} / m,-\boldsymbol{\Omega} \wedge \boldsymbol{\omega}\right)$ is the rate of change of $\boldsymbol{\gamma}$ due to external forces and torques.

The first term on the right hand side of Eq. (10) takes into account the mutual interaction of the ring particles through long-range gravitational forces whose potential is $\phi\left(x_{r}\right)$, being $x_{r}=\left\|\boldsymbol{x}_{1}-\boldsymbol{x}\right\|$ the distance between the centers of two particles. The second term gives the contribution of binary inelastic collisions to the rate of change of $f_{1}$. The relative velocity of two colliding spheres is $\boldsymbol{\xi}_{r}=\boldsymbol{\xi}_{1}-\boldsymbol{\xi}$ and the factor $\left(\boldsymbol{k} \circ \boldsymbol{\xi}_{r}\right)^{+}$restricts collisions to approaching particles pairs. Two comments about the gain part in the collision term are in order. First, the primed values $\gamma^{\prime}$ and $\gamma_{1}^{\prime}$ are the initial velocities of two particles whose final states are $\gamma$ and $\gamma_{1}$, after a collision. The primed states do not coincide with $\gamma^{*}$ and $\gamma_{1}^{*}$, but they can be easily computed as functions of $\gamma$ and $\gamma_{1}$ from Eqs. (7)-(8). Second, the factor $\Lambda$, which is unity in the elastic limit, is defined as $e^{-1} \partial\left(\boldsymbol{\gamma}^{\prime}, \boldsymbol{\gamma}_{1}^{\prime}\right) / \partial\left(\boldsymbol{\gamma}, \boldsymbol{\gamma}_{1}\right)$, $\partial\left(\boldsymbol{\gamma}^{\prime}, \boldsymbol{\gamma}_{1}^{\prime}\right) / \partial\left(\boldsymbol{\gamma}, \boldsymbol{\gamma}_{1}\right)$ being the Jacobian of the transformation relating $\gamma^{\prime}$ and $\gamma_{1}^{\prime}$ to $\gamma$ and $\gamma_{1}$. As a contraction of the pseudo-Liouville equation down to the one-particle level, Eq. (10) is exact, but of little use, since the evolution of $f_{1}$ depends on $f_{2}$, too.

As is well known, in the case of a moderately dense hard sphere fluid in which collisions are elastic and any soft interaction is neglected $\left(\phi\left(x_{r}\right) \equiv 0\right)$, the Enskog theory (Enskog 1922) proposes a way out of this difficulty by assuming that the pair distribution function, $f_{2}(\boldsymbol{x}, \boldsymbol{\gamma}$, $\left.\boldsymbol{x}-a \boldsymbol{k}, \boldsymbol{\gamma}_{1}\right)$, can be expressed as:

$$
\begin{aligned}
f_{2}\left(\boldsymbol{x}, \boldsymbol{\gamma}, \boldsymbol{x}-a \boldsymbol{k}, \boldsymbol{\gamma}_{1}\right)= & Y\left[n\left(\boldsymbol{x}-\frac{a}{2} \boldsymbol{k} \mid t\right)\right] \\
& \times f_{1}(\boldsymbol{x}, \boldsymbol{\gamma} \mid t) f_{1}\left(\boldsymbol{x}-a \boldsymbol{k}, \boldsymbol{\gamma}_{1} \mid t\right)
\end{aligned}
$$

$Y(n)$ being the contact value of the pair correlation function in a hard sphere fluid in uniform equilibrium with density $n . Y(n)$ can be easily obtained from the equation of state (EOS) of the hard sphere fluid (Resibois \& De Leener 1977). In this paper, as in many other previous investigations, $Y(n)$ is computed from the approximate EOS proposed in (Carnahan \& Starling 1969)

$Y(\eta)=\frac{1}{2} \frac{2-\eta}{(1-\eta)^{3}}$ $\eta=\frac{\pi}{6} a^{3} n$ being the volume fraction occupied by the spherical particles.

Although rather crude, the use of Eqs. (11), (12), produces a fairly accurate description of the hard sphere gas as long as the density remains in the validity range of Carnahan-Starling EOS $(0 \leq \eta \leq 0.47)$ and the density does not vary too rapidly on the scale of the molecular diameter $a$. The extension of this simplified treatment of pair correlations to granular fluids is mainly justified by the lack of better theories and requires some caution. Nevertheless, this approach is followed here, on the ground of the good results obtained in previous applications of Enskog kinetic theory (Araki \& Tremaine 1986; Araki 1991). It should be noticed that Eq. (12) will be also used in simulations in which the ring's self-gravity is taken into account. In doing so, it is implicitly assumed that long-range forces do not directly affect short-range correlations, but only act indirectly through modifications of local density.

Neglecting pair correlations in the first term on the right hand side of Eq. (10) leads to the following expression for the gravitational force generated at location $x$ by the ring particles themselves

$\boldsymbol{F}^{\mathrm{sg}}(\boldsymbol{x} \mid t)=m G \int \frac{\rho\left(\boldsymbol{x}_{1} \mid t\right)\left(\boldsymbol{x}_{1}-\boldsymbol{x}\right)}{\left\|\boldsymbol{x}_{1}-\boldsymbol{x}\right\|^{3}} \mathrm{~d}^{3} \boldsymbol{x}_{1}$.

This further simplification is justified because the results presented in this paper are compared with previous calculations in which the treatment of the self-gravitation term was either similar (Salo 1991a) or even more drastically simplified (Wisdom \& Tremaine 1988).

For sake of completeness, a simple estimate of spatial correlation effects on the gravitational force has been performed by taking into account only excluded volume corrections when the density gradient is orthogonal to the ring plane. The results show that spatial correlation corrections give a (limited) contribution when the quantity $\frac{1}{\rho} \frac{\partial \rho}{\partial z} a$ is of unit order, i.e. when the ring is strongly flattened and its thickness is close to $a$. form:

Equation (10) can now explicitly be rewritten in the

$$
\begin{aligned}
& \frac{\partial f_{1}}{\partial t}+\boldsymbol{\xi} \circ \nabla_{\boldsymbol{x}} f_{1}+\frac{\boldsymbol{F}^{\mathrm{tot}}}{m} \circ \nabla_{\boldsymbol{\xi}} f_{1} \\
& -(\boldsymbol{\Omega} \wedge \boldsymbol{\omega}) \circ \nabla_{\boldsymbol{\omega}} f_{1}=C_{\mathrm{E}}\left(f_{1}, f_{1}\right)
\end{aligned}
$$

where the components of the particle acceleration $\frac{\boldsymbol{F}^{\mathrm{tot}}}{m}$ are computed from Eqs. (4) and the collision integral is defined as

$$
\begin{aligned}
C_{\mathrm{E}}\left(f_{1}, f_{1}\right)=a^{2} \int\left\{Y\left[n\left(\boldsymbol{x}+\frac{a \boldsymbol{k}}{2}\right)\right] f_{1}\left(\boldsymbol{x}+a \boldsymbol{k}, \boldsymbol{\gamma}_{1}^{\prime} \mid t\right)\right. \\
\quad \times f_{1}\left(\boldsymbol{x}, \boldsymbol{\gamma}^{\prime} \mid t\right) \Lambda-Y\left[n\left(\boldsymbol{x}-\frac{a \boldsymbol{k}}{2}\right)\right] \\
\left.\quad \times f_{1}\left(\boldsymbol{x}-a \boldsymbol{k}, \boldsymbol{\gamma}_{1} \mid t\right) f_{1}(\boldsymbol{x}, \boldsymbol{\gamma} \mid t)\right\}\left(\boldsymbol{\xi}_{r} \circ \boldsymbol{k}\right)^{+} \mathrm{d} \boldsymbol{\gamma}_{1} \mathrm{~d}^{2} \boldsymbol{k}
\end{aligned}
$$




\subsection{A simplified 1-D equation for the vertical ring structure}

It should now be observed that the linearization of the planet gravitational force term and the dependence of the self-gravity term on relative particle positions makes Eq. (14) invariant upon the transformation:

$$
\begin{aligned}
x^{\prime} & =x-x_{\mathrm{O}^{\prime}}, \\
y^{\prime} & =y+\frac{3 \Omega x_{\mathrm{O}^{\prime}}}{2} t, \\
z^{\prime} & =z, \\
\boldsymbol{\xi}^{\prime} & =\boldsymbol{\xi}+\frac{3 \Omega x_{\mathrm{O}^{\prime}}}{2} \hat{\boldsymbol{y}}
\end{aligned}
$$

which corresponds to describing the particle motion in a reference frame whose origin is $\mathrm{O}^{\prime}$ moving with orbital velocity $\boldsymbol{v}_{\mathrm{O}^{\prime}}=-\frac{3 \Omega x_{\mathrm{O}^{\prime}}}{2} \hat{\boldsymbol{y}}$ with respect to the old reference frame, because of the Keplerian (linearized) differential motion which is locally approximated as a uniform shear flow.

This observation suggests the possibility of looking for solutions of Eq. (14) in which the distribution function appears the same, when observed by a local reference frame translating with velocity $\boldsymbol{v}_{\mathrm{O}^{\prime}}$.

Accordingly, solutions are sought in the form: $f_{1}(x, y, z, \boldsymbol{\xi}, \boldsymbol{\omega} \mid t)=f_{0}\left(z, \boldsymbol{\xi}-\boldsymbol{v}_{\mathrm{O}^{\prime}}, \boldsymbol{\omega} \mid t\right)$. The solution depends on the radial coordinate $x$ implicitly through $\boldsymbol{v}_{\mathrm{O}^{\prime}}$, whereas the dependence on $y$ has been suppressed, because of the further assumption of azimuthal symmetry of the ring. When $f_{0}$ is inserted into Eq. (14), the following kinetic equation is obtained:

$\frac{\partial f_{0}}{\partial t}+c_{z} \frac{\partial f_{0}}{\partial z}+\dot{\boldsymbol{c}} \circ \frac{\partial f_{0}}{\partial \boldsymbol{c}}+\dot{\boldsymbol{\omega}} \circ \frac{\partial f_{0}}{\partial \boldsymbol{\omega}}=C_{\mathrm{E}}\left(f_{0}, f_{0}\right)$

where $\boldsymbol{c}=\boldsymbol{\xi}-\boldsymbol{v}_{\mathrm{O}^{\prime}}, \dot{\boldsymbol{c}}=2 \Omega c_{y} \hat{\boldsymbol{x}}-\frac{\Omega}{2} c_{y} \hat{\boldsymbol{y}}+\left(-\Omega^{2} z+\frac{F_{z}^{\mathrm{sg}}}{m}\right) \hat{\boldsymbol{z}}$ and $\dot{\boldsymbol{\omega}}$ is given by Eq. (3). The collision integral is given by the following expression:

$$
\begin{aligned}
C_{\mathrm{E}}\left(f_{0}, f_{0}\right)= & a^{2} \int\left\{Y\left[n\left(z+\frac{a k_{z}}{2}, t\right)\right]\right. \\
& \times f_{0}\left(z+a k_{z}, \boldsymbol{c}_{\mathbf{1}}^{\prime}, \boldsymbol{\omega}_{\mathbf{1}}^{\prime} \mid t\right) f_{0}\left(z, \boldsymbol{c}^{\prime}, \boldsymbol{\omega}^{\prime} \mid t\right) \Lambda \\
& -Y\left[n\left(z-\frac{a k_{z}}{2}, t\right)\right] f_{0}\left(z-a k_{z}, \boldsymbol{c}_{\mathbf{1}}, \boldsymbol{\omega}_{\mathbf{1}} \mid t\right) \\
& \left.\times f_{0}(z, \boldsymbol{c}, \boldsymbol{\omega} \mid t)\right\}\left(\boldsymbol{c}_{r} \circ \boldsymbol{k}\right)^{+} \mathrm{d} \boldsymbol{c}_{1} \mathrm{~d} \boldsymbol{\omega}_{1} \mathrm{~d}^{2} \boldsymbol{k}
\end{aligned}
$$

where $\boldsymbol{c}_{1}=\boldsymbol{\xi}_{1}+\alpha a k_{x} \hat{\boldsymbol{y}}, \boldsymbol{c}_{r}=\boldsymbol{\xi}_{1}-\boldsymbol{c}, \boldsymbol{c}_{1}^{\prime}=\boldsymbol{\xi}_{1}^{\prime}-\alpha a k_{x} \hat{\boldsymbol{y}}$, $\alpha=-\frac{3}{2} \Omega$. Equation (17) describes one-dimensional solutions in which all flow field macroscopic properties, but not the uniformly shearing bulk velocity $y$-component, are uniform on each plane parallel to the equatorial plane and vary only along the $z$ direction.

The assumed ring homogeneity on the $x y$ planes causes the horizontal components of the self-gravity force to vanish. The vertical component $F_{z}^{\mathrm{sg}}$ is given by Eq. (13) which simplifies to

$$
F_{z}^{\mathrm{sg}}(z \mid t)=2 \pi m G \int_{-\infty}^{+\infty} \rho\left(z_{1} \mid t\right) \frac{\left(z_{1}-z\right)}{\left|z_{1}-z\right|} \mathrm{d} z_{1} .
$$

\section{The numerical method}

The statistical particle method used to obtain numerical solutions of Eq. (17) has been proposed and described in Frezzotti (1997) in connection with the original Enskog equation for the dense hard sphere gas. The method is based on the representation of the distribution function as a superposition of $\delta$ functions:

$f_{1}(\boldsymbol{x}, \boldsymbol{\gamma} \mid t)=\mathcal{W} \sum_{i=1}^{N_{\mathrm{p}}} \delta\left(\boldsymbol{x}-\boldsymbol{x}_{i}(t)\right) \delta\left(\boldsymbol{\gamma}-\boldsymbol{\gamma}_{i}(t)\right)$.

In Eq. (20) each term $\delta\left(\boldsymbol{x}-\boldsymbol{x}_{i}\right) \delta\left(\boldsymbol{\gamma}-\boldsymbol{\gamma}_{i}\right)$ represents the contribution of the $i$ th particle out of the $N_{\mathrm{p}}$ used in the calculation. It should be noticed that, in general, $N_{\mathrm{p}}$ is not equal to the real number of particles present in the simulated flow field and the weight $\mathcal{W}$, equal to the ratio of the number of real to the number of simulation particles, accounts for that.

The time evolution of $f$ across the interval $[t, t+\Delta t]$ is obtained by a first order time-splitting scheme consisting of two steps. In the first, particles move through the computational domain without colliding according to the equation:

$\frac{\partial f_{1}}{\partial t}+\boldsymbol{\xi} \circ \nabla_{\boldsymbol{x}} f_{1}+\frac{\boldsymbol{F}^{\mathrm{tot}}}{m} \circ \nabla_{\boldsymbol{\xi}} f_{1}-(\boldsymbol{\Omega} \wedge \boldsymbol{\omega}) \circ \nabla_{\boldsymbol{\omega}} f_{1}=0$

which, in terms of particle positions and velocities reads:

$\frac{\mathrm{d} \boldsymbol{x}_{i}}{\mathrm{~d} t}=\boldsymbol{\xi}_{i} \quad \frac{\mathrm{d} \boldsymbol{\xi}_{i}}{\mathrm{~d} t}=\frac{\boldsymbol{F}_{i}^{\mathrm{tot}}}{m} \quad \frac{\mathrm{d} \boldsymbol{\omega}_{i}}{\mathrm{~d} t}=-\boldsymbol{\Omega} \wedge \boldsymbol{\omega}_{i}$

Any suitable finite difference scheme can be used to obtain particle positions and provisional velocities at time $t+$ $\Delta t$ from Eq. (22). Once the first step is completed, the variation on $f_{1}$ induced by the collision term on the right hand side of Eq. (14) is computed from the equation:

$\frac{\partial f_{1}}{\partial t}=C_{\mathrm{E}}\left(f_{1}, f_{1}\right)$

In this second step each particle keeps the position reached at the end of the previous step, whereas particle velocities $\gamma_{i}$ are updated according to a probabilistic method which provides an estimate for the overall collision rate in the computational domain and selects collision pairs according to Eq. (23).

We observe that the collision rate of simulation particles is

$N_{\mathrm{c}}=\frac{1}{2} \sum_{i=1}^{N_{\mathrm{p}}} \nu_{i}$

where

$$
\begin{aligned}
\nu_{i}= & a^{2} \int Y\left[n\left(\boldsymbol{x}_{i}-\frac{a}{2} \boldsymbol{k}\right)\right] \\
& \times f_{1}\left(\boldsymbol{x}_{i}-a \boldsymbol{k}, \boldsymbol{\gamma}_{1}\right)\left[\left(\boldsymbol{\xi}_{1}-\boldsymbol{\xi}_{i}\right) \circ \boldsymbol{k}\right]^{+} \mathrm{d} \boldsymbol{\gamma}_{1} \mathrm{~d}^{2} \boldsymbol{k} .
\end{aligned}
$$

To obtain the collision probability of two simulation particles the computational domain is divided into a number of cells whose size must be small enough to resolve 
the flow field gradients. Within each cell, the distribution function (20) is spatially regularized and it takes the following form:

$\tilde{f}_{1}(\boldsymbol{x}, \gamma \mid t)=\frac{n_{m}}{N_{m}} \sum_{j=1}^{N_{m}} \delta\left(\gamma-\gamma_{m j}\right), \quad \boldsymbol{x} \in \mathcal{C}_{m}$

In Eq. (26) $n_{m}$ and $N_{m}$ are the real average number density and the number of simulation particles in the $m$ th cell $\mathcal{C}_{m}$ respectively, whereas $\gamma_{m j}$ is the velocity of particle $j$ in $\mathcal{C}_{m}$. Substituting expression (26) into Eqs. (24), (25) yields the following result:

$$
\begin{aligned}
N_{c}= & \frac{1}{2} \sum_{i=1}^{N_{\mathrm{p}}} \sum_{m=1}^{M_{i}} \sum_{j=1}^{N_{m}} \nu_{i m j} \\
\nu_{i j m}= & a^{2} \frac{n_{m}}{N_{m}} \int_{\mathcal{S}_{i m}} Y\left[n\left(\boldsymbol{x}_{i}-\frac{a}{2} \boldsymbol{k}\right)\right] \\
& \times\left[\left(\boldsymbol{\xi}_{m j}-\boldsymbol{\xi}_{i}\right) \circ \boldsymbol{k}\right]^{+} \mathrm{d}^{2} \boldsymbol{k}
\end{aligned}
$$

being $M_{i}$ the number of cells containing a portion of the sphere having radius $a$ and center $\boldsymbol{x}_{i}$, and $\mathcal{S}_{i m}$ the region of the unit sphere where $\boldsymbol{x}_{i}-a \boldsymbol{k} \in \mathcal{C}_{m}$. Although correct, the direct evaluation of $N_{\mathrm{c}}$ from Eq. (27) would require a computational effort proportional to $N_{\mathrm{p}}^{2}$. This difficulty is circumvented by the adoption of a majorant collision frequency scheme (Koura 1986) in which $N_{\mathrm{c}}$ is estimated by a stochastic algorithm whose computational effort grows linearly with $N_{\mathrm{p}}$. An upper bound $\bar{\nu}_{i j m}$ for each $\nu_{i j m}$ is easily obtained as

$\nu_{i j m} \leq \bar{\nu}_{i j m}=a^{2} \frac{A_{i} C_{i}}{N_{m}} \int_{\mathcal{S}_{i m}} \mathrm{~d}^{2} \boldsymbol{k}$

where $A_{i}$ and $C_{i}$ satisfy the following inequalities:

$n_{m} Y\left[n\left(\boldsymbol{x}_{i}-\frac{a}{2} \boldsymbol{k}\right)\right] \leq A_{i}, \quad \forall \boldsymbol{k} \in \mathcal{S}_{i m}, \quad m=1 \ldots M_{i}$

$\left\|\boldsymbol{\xi}_{m j}-\boldsymbol{\xi}_{i}\right\| \leq C_{i}, \forall m j$.

An upper bound $\bar{N}_{\mathrm{c}}$ for the collision rate immediately follows from Eq. (29)

$\bar{N}_{\mathrm{c}}=\frac{1}{2} \sum_{i=1}^{N_{\mathrm{p}}} \sum_{m=1}^{M_{i}} \sum_{j=1}^{N_{m}} \bar{\nu}_{i m j}=\sum_{i=1}^{N_{\mathrm{p}}} 2 \pi a^{2} A_{i} C_{i}$.

The method consists of replacing the real collision rate $N_{\mathrm{c}}$ with $\bar{N}_{\mathrm{c}}$ which is easier to obtain. However, the real collision rate will not be altered because the extra collisions will be false collisions which do not change the velocities of colliding pairs.

According to Eq. (32), the probability that particle $i$ is selected for a collision (either real or false) is

$\bar{p}_{i}=\frac{2 \pi a^{2} A_{i} C_{i}}{\bar{N}_{\mathrm{c}}}$.

Once particle $i$ has been selected, the probability that it collides with a particle in cell $m$ is

$\bar{p}_{i m}=\frac{\sum_{j=1}^{N_{m}} \bar{\nu}_{i j m}}{\sum_{m=1}^{M_{i}} \sum_{j=1}^{N_{m}} \bar{\nu}_{i j m}}=\frac{1}{4 \pi} \int_{\mathcal{S}_{i m}} \mathrm{~d}^{2} \boldsymbol{k}$.
Equation (34) shows that $\bar{p}_{i m}$ equals the probability that, drawing a random vector $\boldsymbol{k}$ on the unit sphere, the position $\boldsymbol{x}_{i}-a \boldsymbol{k}$ of the collision partner is in cell $\mathcal{C}_{m}$. The probability that particle $j$ out of the $N_{m}$ particles in $\mathcal{C}_{m}$ is selected for a collision is

$\bar{p}_{i j m}=\frac{\bar{\nu}_{i j m}}{\sum_{j=1}^{N_{m}} \bar{\nu}_{i j m}}=\frac{1}{N_{m}}$.

The above result implies that the collision partner of particle $i$ can be chosen at random among the particles in cell $\mathcal{C}_{m}$. The partner selection rules are based on the modified collision frequencies $\bar{\nu}_{i j m}$ which include false collisions. The probability $p_{i j m}$ that the collision between particles $i$ and $m j$ is real is given by the following expressions:

$\begin{aligned} p_{i j m} & =\frac{\nu_{i j m}}{\bar{\nu}_{i j m}}=\frac{\int_{\mathcal{S}_{m i}} \phi(\boldsymbol{k}) \mathrm{d}^{2} \boldsymbol{k}}{\int_{\mathcal{S}_{m i}} \mathrm{~d}^{2} \boldsymbol{k}} \\ \phi(\boldsymbol{k}) & =\frac{n_{m} Y\left[n\left(\boldsymbol{x}_{i}-\frac{a}{2} \boldsymbol{k}\right)\right]\left[\left(\boldsymbol{\xi}_{m j}-\boldsymbol{\xi}_{i}\right) \circ \boldsymbol{k}\right]^{+}}{A_{i} C_{i}} .\end{aligned}$

Since $0 \leq \phi(\boldsymbol{k}) \leq 1, p_{i j m}$ is also equal to the probability that a random fraction $r_{\mathrm{f}}$ is less than $\phi(\boldsymbol{k})$, being $\boldsymbol{k}$ uniformly distributed in $\mathcal{S}_{m i}$. If the collision is accepted as real, then the velocities of collision partners are changed according to Eqs. (7), (8).

\section{Numerical results}

The numerical method briefly described in the previous section has been applied to obtain stationary solutions of Eq. (17). If the basic quantities are made non-dimensional by using the particle diameter $a$ as unit length, $\Omega^{-1}$ as unit time and the particle mass $m$ as unit mass, it can be easily shown that steady solutions do depend only on the two dimensionless parameters

$\tau=\frac{\pi a^{2}}{4} \int_{-\infty}^{+\infty} n(z) \mathrm{d} z \quad G_{\mathrm{s}}=\frac{8 G m}{\Omega^{2} a^{3}}$

once the collision restitution coefficients $e$ and $\beta$ have been fixed. The optical depth $\tau$ is proportional to the ring surface density, whereas the parameter $G_{\mathrm{S}}$ is a measure of the importance of the self-gravity term.

Since the numerical method is intrinsically unsteady, a stationary solution is obtained as the long time limit of a properly initialized unsteady solution. In this particular problem the onset of stationary conditions is monitored through the value of the total kinetic energy of the system, which finally reaches a constant value as a result of the balancing between viscous heating and collisional energy dissipation.

After decaying of the initial transient, the flow field properties are obtained by sampling and time averaging particle positions and velocities over a suitable time interval $t_{\text {samp }}$. The results described below have been obtained by using 40000 simulation particles. It is difficult to give a precise and problem-independent estimate of the minimal number of particles required for an accurate simulation. 
However, the results are fairly insensitive to $N_{\mathrm{p}}$, provided the domain contains at least 10 computational particles per cell and the sample size (or $N_{\mathrm{p}} t_{\mathrm{samp}}$ ) is kept fixed. The choice of the number of simulation particles requires some care only at high density, because strong statistical density fluctuations in a simulation with low $N_{\mathrm{p}}$ might locally bring $\eta$ close to one where Eq. (12) has an unphysical singularity. The time step $\Delta t$ to be used in the simulation is determined by the condition

$\Delta t=\min \left(\Delta t_{\mathrm{ff}}, \Delta t_{\text {coll }}\right)$

where $\Delta t_{\mathrm{ff}}$ is the time step required for an accurate treatment of the free flight step described by Eqs. (22) and $\Delta t_{\text {coll }}$ is the time step to be used in the collision step. $\Delta t_{\text {coll }}$ can be estimated from the inequality $\Delta t_{\text {coll }} \bar{\nu} \ll 1$, $\bar{\nu}$ being the highest value of the collision frequency in the computational domain.

\subsection{Simulations without self-gravity effects}

In the first group of simulations self-gravity effects have been neglected $\left(G_{\mathrm{S}}=0\right)$ and an investigation of the ring stability has been performed as a preliminary accuracy test. Actually, as pointed out by Goldreich \& Tremaine (1978) in their investigation of dilute rings, a steady flow field does not exist for an arbitrary choice of the optical depth $\tau$ and restitution coefficient $e$, since energy production by viscous heating must be exactly balanced by collisional energy dissipation. In the dilute ring approximation, where non-local collisional energy and momentum transfer are neglected, balancing occurs only on a curve on the $(\tau, e)$ plane. However, when non-local interaction effects are included, the curve becomes the upper boundary of the ring stability region and steady solutions are found for any $(\tau, e)$ point below it.

A few points on the critical line have been calculated by the DSMC solution of Eq. (17) and compared with the approximate theoretical Goldreich-Tremaine curve $((1-$ $\left.\left.e^{2}\right)\left(1+\tau^{2}\right) \approx 0.6\right)$ and the recent MD results in (Schmidt et al. 1999). Each point required a few computations with a fixed value of $\tau$ and different values of $e$. The total kinetic energy of the system was monitored for a long time to detect either the onset of a steady state or an unstable behavior. The final value of $e$ was determined as the lowest in a pair $\left(e_{\mathrm{s}}, e_{\mathrm{u}}\right)$ of values such that $e_{\mathrm{s}}$ led to steady conditions, $e_{\mathrm{u}}$ was judged to lead to ring instability and $e_{\mathrm{u}}-e_{\mathrm{s}}=0.0025$. As shown in Fig. 1a, the agreement between the present results and MD simulations is excellent.

The effects of the addition of surface roughness on the ring stability have also been investigated by a limited number of simulations whose results are presented in Fig. 1b.

As expected, tangential friction enhances energy dissipation, as predicted by Eq. (9). Accordingly, critical restitution coefficients $e$ are shifted upward for any given $\tau$. The $\beta-e$ curve for $\tau=0.1$ is very close to the moment method predictions by Araki (1991) in the limit $\tau \rightarrow 0$. A significant difference between DSMC and moment method calculations is found only in the vicinity of (a)
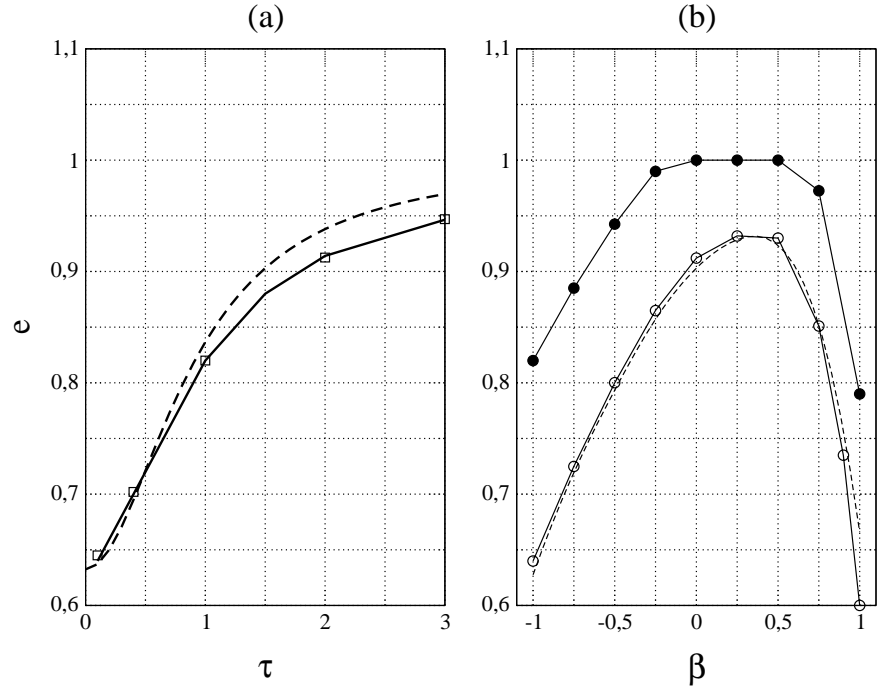

Fig. 1. a) Ring stability curves in $\tau-e$ plane. The dotted line represents Goldreich \& Tremaine (1978) relationship; solid line represents DSMC results; square symbols represent MD simulations results from (Schmidt et al. 1999). b) Ring stability curves in $\beta-e$ plane at $\tau=0.1$ (circles) and $\tau=1.0$ (squares); the dotted line represents the limit curve $\tau \rightarrow 0$ obtained by Araki (1991).

$\beta=1$ (perfectly rough spheres), where the former method predicts a lower critical $e$, which, however, turns out to be in agreement with a previous calculation (Araki 1988) where non-local collisions effects were neglected.

Particular attention has been devoted to reproducing the MD results described in the paper (Wisdom \& Tremaine 1988) (hereafter WT), in the case of smooth spheres $(\beta=-1)$ and constant normal restitution coefficient $e=0.5$. The optical depth $\tau$, the only remaining free parameter, has been varied in the range $[0.1,2.2]$. Two examples of vertical density profiles, normalized to the density maximum, are shown in Fig. 2. The corresponding optical depth values are 1.0 and 2.0, respectively. The agreement with MD results is very good in both cases and it is not only limited to the shape of the density profiles, as shown by Fig. 3 where the volume fraction $\eta$ in the center of the ring is displayed as a function of $\tau$. It is worth noting that a similar accuracy degree has been achieved by Araki \& Tremaine (1986) (hereafter AT) in predicting the central volume fraction $\eta(0)$ by a moment method solution of the Enskog equation. However, the agreement of their density profiles with MD results was only qualitative, being based on a Gaussian Ansatz for the density profile.

As is well known, the velocity distribution function iso-surfaces in sheared granular media take an approximately elliptic shape. Therefore, it is rather natural to give a global description of $f_{0}$ through the parameters that identify the associated velocity ellipsoid: the orientation of the ellipsoid principal axes and the velocity dispersions along the principal directions. Such characteristics can be obtained at each spatial location $z$ by looking for 

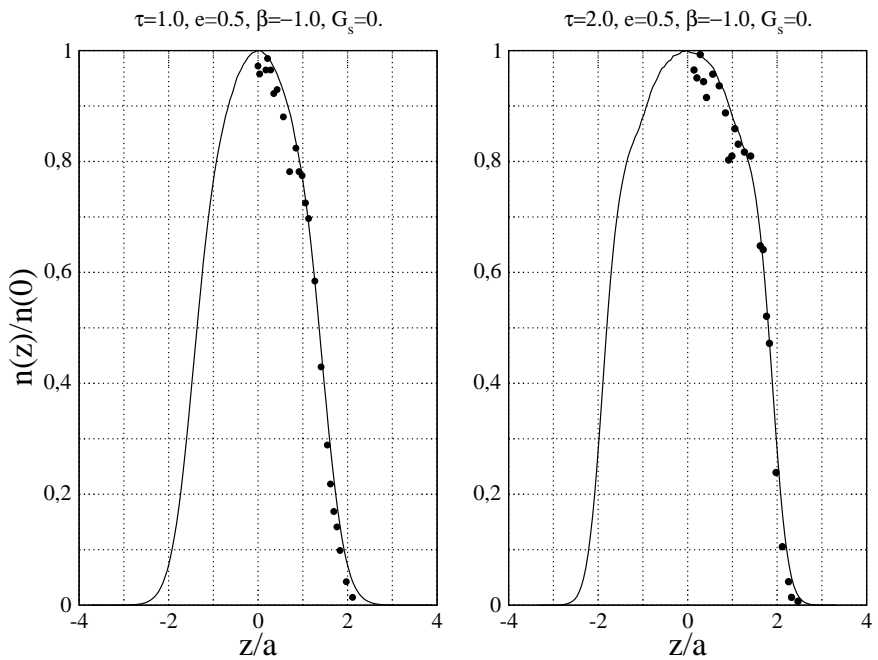

Fig. 2. Normalized density profiles. Solid curve: DSMC; circles: MD simulations from WT. Graph a): $e=0.5, \beta=-1, G_{\mathrm{s}}=0$, $\tau=1.0$; graph b): $e=0.5, \beta=-1, G_{\mathrm{s}}=0, \tau=2.0$.

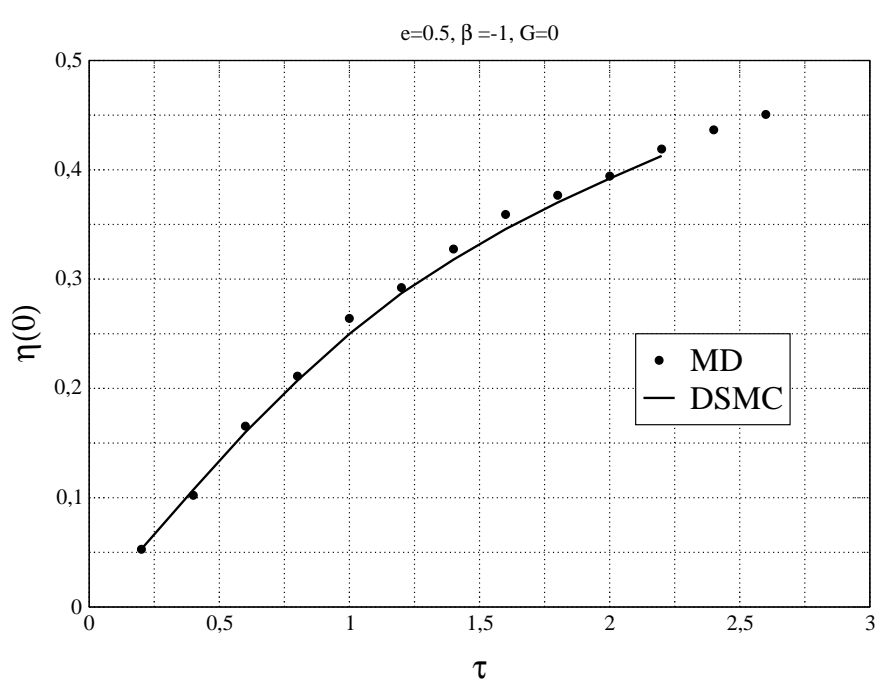

Fig. 3. The volume fraction $\eta(0)$ in the center of the ring as a function of $\tau$. Solid curve: DSMC; circles: MD simulations from WT.

the eigenvector and eigenvalues of the kinetic part of the stress tensor

$\mathrm{P}^{\mathrm{k}}(\boldsymbol{x})=m \int \boldsymbol{c} \boldsymbol{c} f_{1}(\boldsymbol{x}, \boldsymbol{\gamma}) \mathrm{d} \boldsymbol{\gamma}$

$\boldsymbol{c}=\boldsymbol{\xi}-\boldsymbol{u}(\boldsymbol{x})$ being the peculiar velocity and $\boldsymbol{u}$ the mean velocity. By symmetry arguments, it turns out that the vertical direction is a principal direction; the remaining two mutually orthogonal principal axes lie on the equatorial plane, but they are generally rotated by an angle $\delta(z)$ with respect to the radial and azimuthal axes $\hat{\boldsymbol{x}}$ and $\hat{\boldsymbol{y}}$. Height-averaged values of the tilt angle $\bar{\delta}$ as a function of $\tau$ are given in Fig. 4 both for DSMC and for MD simulations of WT. The DSMC $\bar{\delta}$ has been computed from the eigenvectors of the height-averaged kinetic stress tensor $\overline{\mathrm{Pk}^{\mathrm{k}}}$. In this case the more refined DSMC solutions of the kinetic equation do not show any improvement over the moment method predictions of AT which are very close

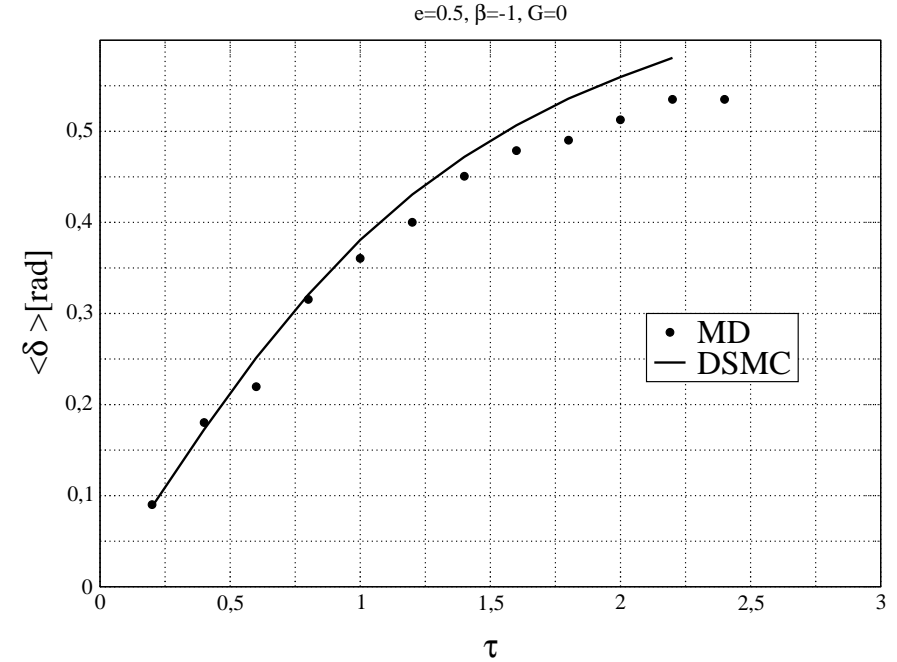

Fig. 4. Height averaged tilt angle $\bar{\delta}$ as a function of $\tau$. Solid curve: DSMC; circles: MD simulations from WT.

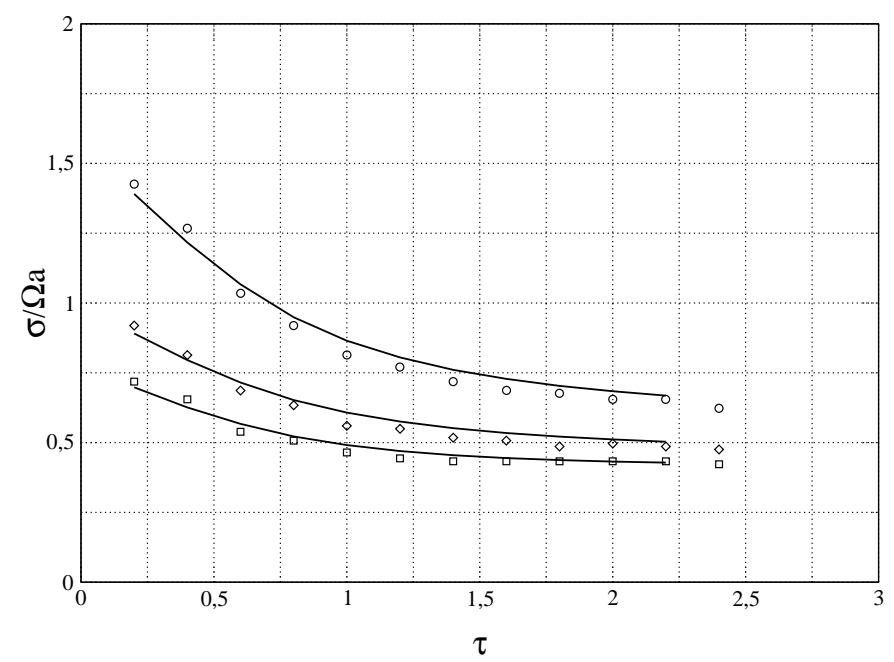

Fig. 5. Height averaged velocity dispersions along the principal axes of the velocity ellipsoid. Values normalized to $a \Omega$. Solid curves: DSMC; symbols MD simulations from WT; circles: $\overline{\sigma_{x}}$, squares: $\overline{\sigma_{y}}$, diamonds: $\overline{\sigma_{z}}$

to the present calculations and deviate from MD data at high optical depths.

The height-averaged velocity dispersions along the principal axes $\overline{\sigma_{x}}, \overline{\sigma_{y}}$ and $\overline{\sigma_{z}}$ are shown in Fig. 5 as solid lines. The corresponding MD values, marked by symbols, are very close to DSMC results; the largest deviation (about $8 \%$ ) occurs at $\tau=1$ for $\overline{\sigma_{z}}$, but the average deviation does not exceed $4 \%$, thus showing a slightly better agreement with MD simulations than AT's moment method.

In addition to the global height-averaged quantities shown above, spatial profiles of the stress tensor components $\mathrm{P}$ and heat flux $\boldsymbol{q}$ have also been calculated.

Both $\mathrm{P}$ and $\boldsymbol{q}$ consist of a kinetic and a collisional contribution

$P=P^{k}+P^{c}$ 
$\boldsymbol{q}=\boldsymbol{q}^{\mathrm{k}}+\boldsymbol{q}^{\mathrm{c}}$

The kinetic contribution $\mathrm{P}^{\mathrm{k}}$ to the total stress tensor has already been defined by Eq. (40), whereas the collisional contribution is (Cercignani \& Lampis 1988; Goldshtein \& Shapiro 1995)

$$
\begin{aligned}
\mathrm{P}^{\mathrm{c}}= & -m \frac{a^{2}}{2} \int \mathrm{d} \boldsymbol{\gamma}_{1} \mathrm{~d} \boldsymbol{\gamma}_{2} \mathrm{~d}^{2} \boldsymbol{k} \int_{0}^{a} \mathrm{~d} \lambda \\
& \times\left(\boldsymbol{\xi}_{1}^{*}-\boldsymbol{\xi}_{1}\right) \boldsymbol{k} Y\left[n\left(\boldsymbol{x}+\lambda \boldsymbol{k}-\frac{a}{2} \boldsymbol{k}\right)\right] \\
& \times f_{1}\left(\boldsymbol{x}+\lambda \boldsymbol{k}-a \boldsymbol{k}, \boldsymbol{\gamma}_{1}, t\right) \\
& \times f_{1}\left(\boldsymbol{x}+\lambda \boldsymbol{k}, \boldsymbol{\gamma}_{2}, t\right)\left(\boldsymbol{\xi}_{r} \circ \boldsymbol{k}\right)^{+} .
\end{aligned}
$$

As far as the heat flux is concerned, we have

$$
\begin{aligned}
\boldsymbol{q}^{\mathrm{k}}= & \frac{m}{2} \int \boldsymbol{c}^{2} \boldsymbol{c} f_{1}(\boldsymbol{x}, \boldsymbol{\gamma} \mid t) \mathrm{d} \boldsymbol{\gamma} \\
\boldsymbol{q}^{\mathrm{c}}= & \frac{m a^{2}}{8} \int \mathrm{d} \boldsymbol{\gamma}_{1} \mathrm{~d} \boldsymbol{\gamma}_{2} \mathrm{~d}^{2} \boldsymbol{k} \int_{0}^{a} \mathrm{~d} \lambda \boldsymbol{k}\left[\left(\boldsymbol{c}_{1}^{* 2}-\boldsymbol{c}_{1}^{2}\right)\right. \\
& \left.-\left(\boldsymbol{c}_{2}^{* 2}-\boldsymbol{c}_{2}^{2}\right)\right] Y\left[n\left(\boldsymbol{x}+\lambda \boldsymbol{k}-\frac{a}{2} \boldsymbol{k}\right)\right] \\
& \times f_{1}\left(\boldsymbol{x}+\lambda \boldsymbol{k}-a \boldsymbol{k}, \boldsymbol{\gamma}_{1}, t\right) \\
& \times f_{1}\left(\boldsymbol{x}+\lambda \boldsymbol{k}, \boldsymbol{\gamma}_{2}, t\right)\left(\boldsymbol{\xi}_{r} \circ \boldsymbol{k}\right)^{+}
\end{aligned}
$$

$\mathrm{P}$ and $\boldsymbol{q}$ appear in the balance equations of momentum and energy which, in the present steady and one-dimensional problem, take the form

$$
\begin{aligned}
& \frac{\mathrm{d} P_{z z}}{\mathrm{~d} z}=-\rho \Omega^{2} z+F_{z}^{\mathrm{sg}} \\
& \frac{\mathrm{d} q_{z}}{\mathrm{~d} z}+\alpha P_{x y}=D^{\mathrm{c}} .
\end{aligned}
$$

The general expression for the energy dissipated by collisions per unit volume and time $D^{\mathrm{c}}$ is

$$
\begin{aligned}
D^{\mathrm{c}}(\boldsymbol{x})= & \frac{m a^{2}}{4} \int \mathrm{d} \boldsymbol{\gamma}_{1} \mathrm{~d} \boldsymbol{\gamma}_{2} \mathrm{~d}^{2} \boldsymbol{k}\left(\boldsymbol{\xi}_{1}^{* 2}+\boldsymbol{\xi}_{2}^{* 2}\right. \\
& \left.-\boldsymbol{\xi}_{1}^{2}-\boldsymbol{\xi}_{2}^{2}\right) Y\left[n\left(\boldsymbol{x}-\frac{a}{2} \boldsymbol{k}\right)\right] \\
& \times f_{1}\left(\boldsymbol{x}, \boldsymbol{\gamma}_{1}\right) f_{1}\left(\boldsymbol{x}-a \boldsymbol{k}, \boldsymbol{\gamma}_{2}\right)\left(\boldsymbol{\xi}_{r} \circ \boldsymbol{k}\right)^{+}
\end{aligned}
$$

The meaning of Eqs. (46), (47) is straightforward. The first one states that fluid elements are in mechanical equilibrium, the total vertical component of the gravitational force being compensated by the gradient of the $P_{z z}$ component of P. Equation (47) states that energy losses by inelastic collisions are balanced by energy produced by viscous heating and heat flux.

The balance equations can be used to assess the consistency, if not the accuracy, of the numerical solutions. Vertical momentum balance is illustrated in Fig. 6 in a case where the optical depth has been set equal to 1.0 and self-gravity is also present.

The analysis of the energy equation is more interesting, as shown by Figs. 7 and 8 which present the two extreme cases $\tau=0.2$ and $\tau=2.0$, respectively. In both cases the derivative of the heat flux is very close to the difference between energy dissipation and energy production, as shown

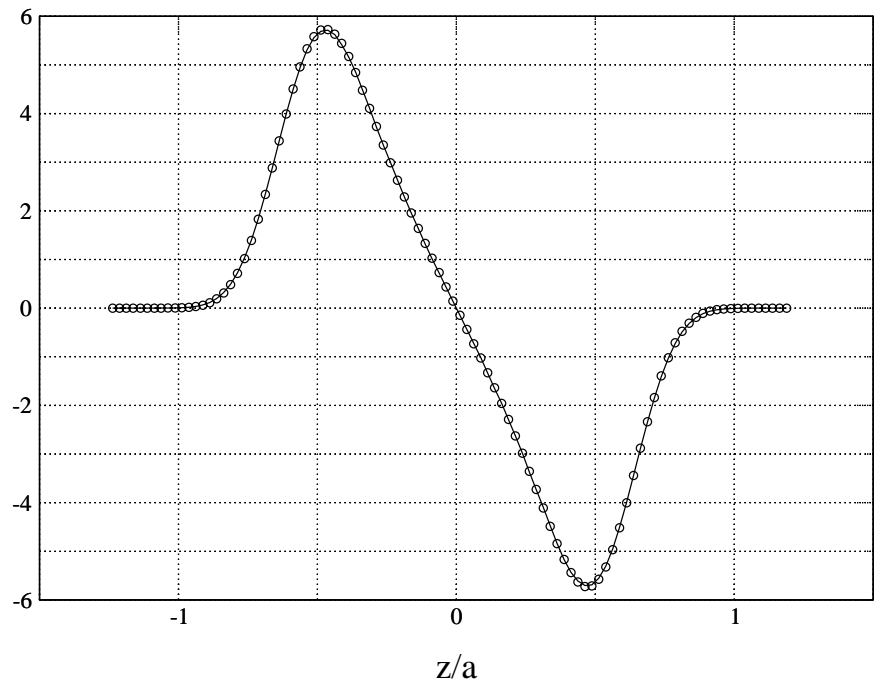

Fig. 6. Vertical momentum balance, $e=0.5, \beta=-1, G_{\mathrm{s}}=$ 7.4, $\tau=1.0$. Solid line, $\frac{\mathrm{d} P_{z z}}{\mathrm{~d} z} ; \circ,-\rho \Omega^{2} z+F_{z}^{\mathrm{sg}}$.

in Figs. 7b and 8b. The intensities of energy gain and loss rates are close for any $z$. This implies that ring layers are approximately thermally insulated. Heat conduction plays a limited role which is more relevant at low optical depth. Actually, if the ring center is considered, then it is found that $\mathrm{d} q_{z} / \mathrm{d} z$ amounts to about $9 \%$ of $\alpha P_{x y}$ at $\tau=0.2$ but decreases to about $3 \%$ at $\tau=2.0$. It is also to be noticed that $q_{z}$, although not monotonic, is an odd function of $z$. Heat flows from the outer part of the ring toward the center where the dissipation rate is higher than the viscous energy production. The sign of $q_{z}$ is consistent with the $U$ shape of the granular temperature profiles which are even functions of $z$ with a minimum on the equatorial plane.

Unfortunately, it is not possible to make a direct comparison of the stress tensor or heat flux components computed by DSMC with similar MD results which seem to be absent from the literature. However, the height-integrated DSMC $P_{x y}$ component can be used to obtain an effective height-averaged dynamic viscosity $\bar{\mu}$ from the relationship $\int_{-\infty}^{+\infty} P_{x y}(z) \mathrm{d} z=\bar{\mu} \alpha$.

Introducing the height-averaged kinematic viscosity $\bar{\nu}=$ $\bar{\mu} / \sigma$, where $\sigma$ is the surface density, the following relationship is easily derived

$\tau \bar{\nu}=\frac{\pi a^{2}}{6 \Omega} \int_{-\infty}^{+\infty} P_{x y}(z) \mathrm{d} z$.

If separately applied to $P_{x y}^{\mathrm{k}}$ and $P_{x y}^{\mathrm{c}}$, the equation above can also be used to obtain the kinetic and collisional contributions, $\tau \overline{\nu^{\mathrm{k}}}$ and $\tau \overline{\nu^{\mathrm{c}}}$, to the overall dynamic viscosity. The DSMC predictions for these quantities are displayed in Fig. 9 where they are also compared to MD results.

The comparison shows that there is very good agreement between the two methods as far as the kinetic contribution is concerned hence, the kinetic theory prediction of the total $\tau \bar{\nu}$ is slightly above the MD data because of the overestimation of the collisional contribution at high $\tau$. 

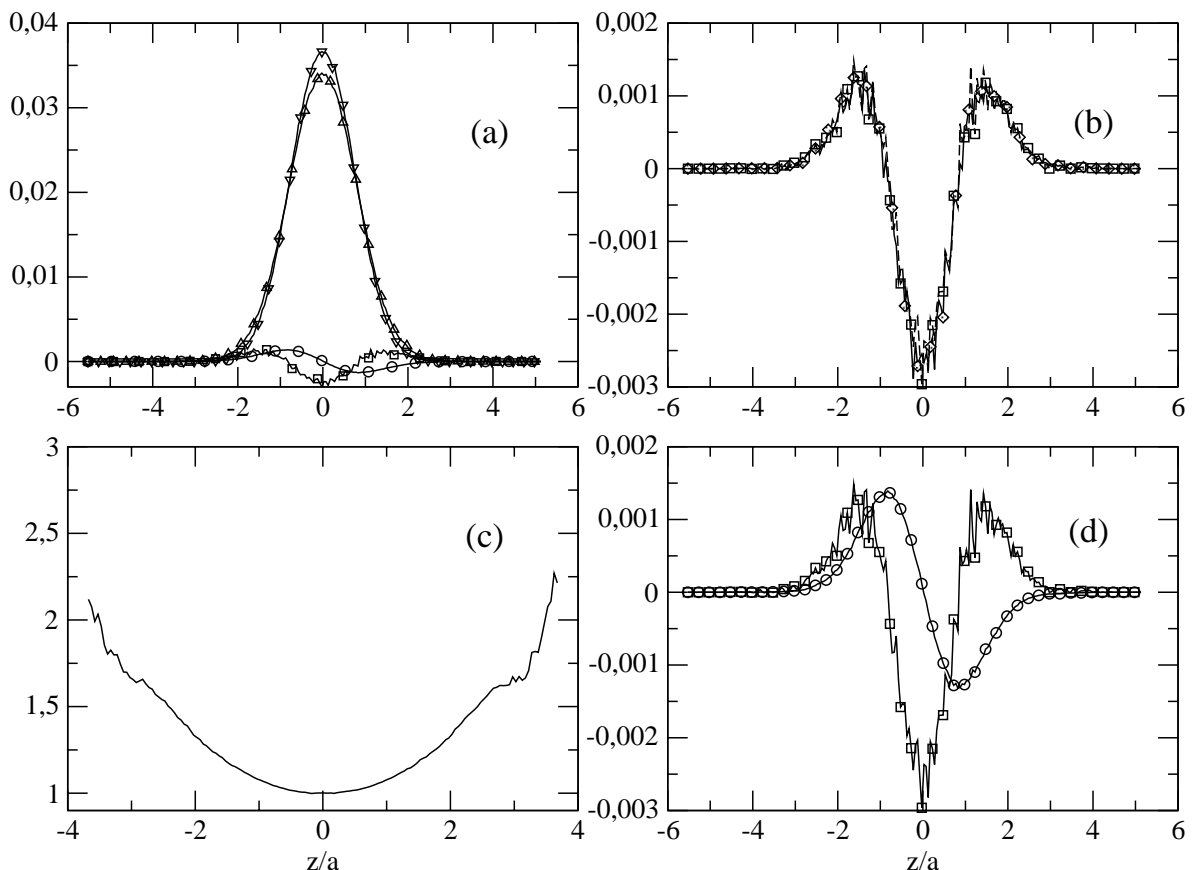

Fig. 7. Vertical thermal balance, $e=0.5, \beta=-1, G_{s}=0, \tau=0.2$. Graph a): $\nabla,\left|D^{\mathrm{c}}(z)\right| ; \triangle,\left|\alpha P_{x y}(z)\right| ; \circ, q_{z}(z) ; \square, \mathrm{d} q_{z} / \mathrm{d} z$. Graph b): $\diamond, D^{\mathrm{c}}(z)-\alpha P_{x y}(z) ; \square, \mathrm{d} q_{z} / \mathrm{d} z$. Graph c): solid line, $T / a \Omega^{2}$. Graph d): $\circ, q_{z}(z) ; \square, \mathrm{d} q_{z} / \mathrm{d} z$.
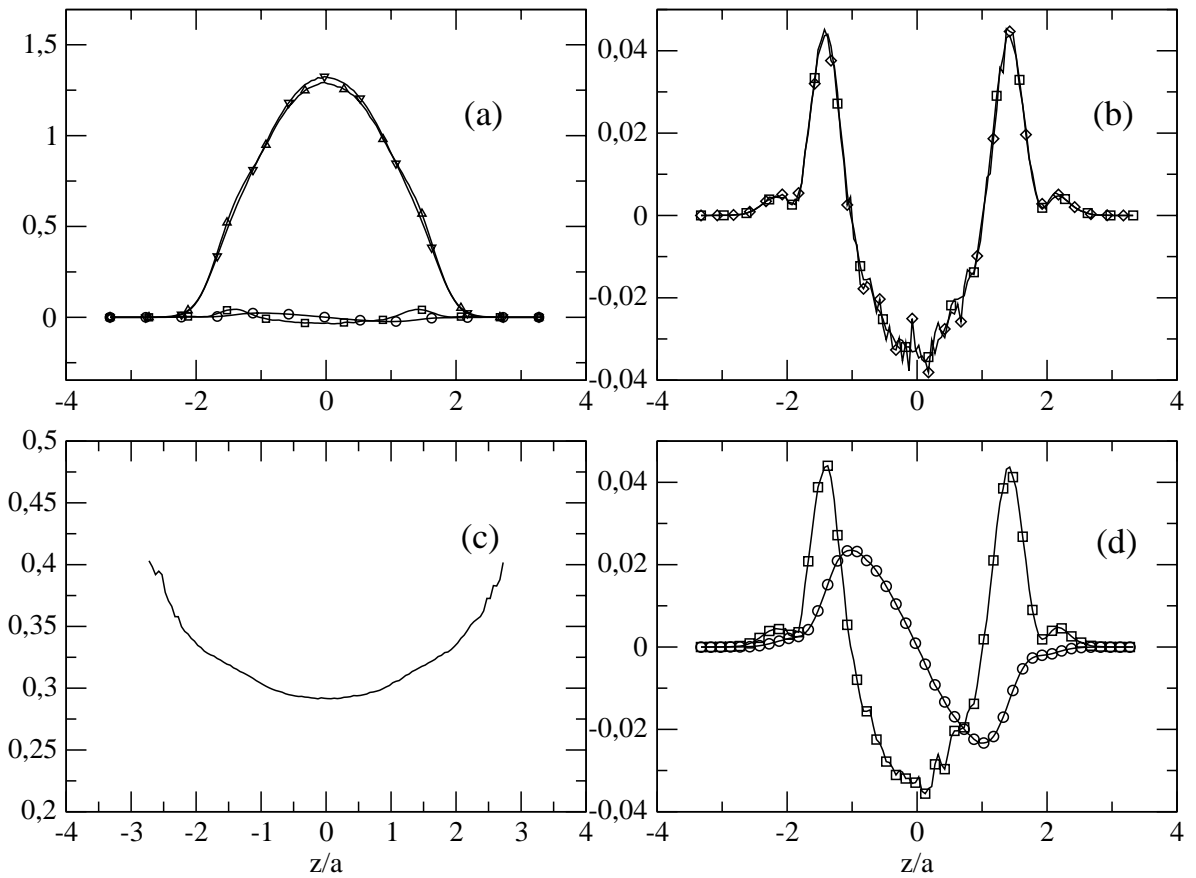

Fig. 8. Vertical thermal balance, $e=0.5, \beta=-1, G_{s}=0, \tau=2.0$. Labeling of graphs and curves is the same as in the previous figure.

\subsection{Simulations with self-gravity effects}

As anticipated in Sect. 2, the effects of self-gravity have been investigated by computing the vertical gravity field from Eq. (19). The DSMC results have been compared with data taken from three previous investigations. The first source is the already-cited WT paper, where selfgravity was simulated by enhancing the frequency of vertical oscillations in Eq. (4) by a factor of 3.6, as a result of the estimated density of Saturn's B-ring. In applying this method, which would be correct if the vertical density profile were flat, it was further assumed that the enhancement factor was independent of the optical depth, thus overestimating self-gravity effects at low $\tau$ 's.

The second source is MD simulations by Salo (Salo 1991a, 1991b) who adopted the same self-consistent approach used here to compute $F_{z}^{\mathrm{sg}}$. 


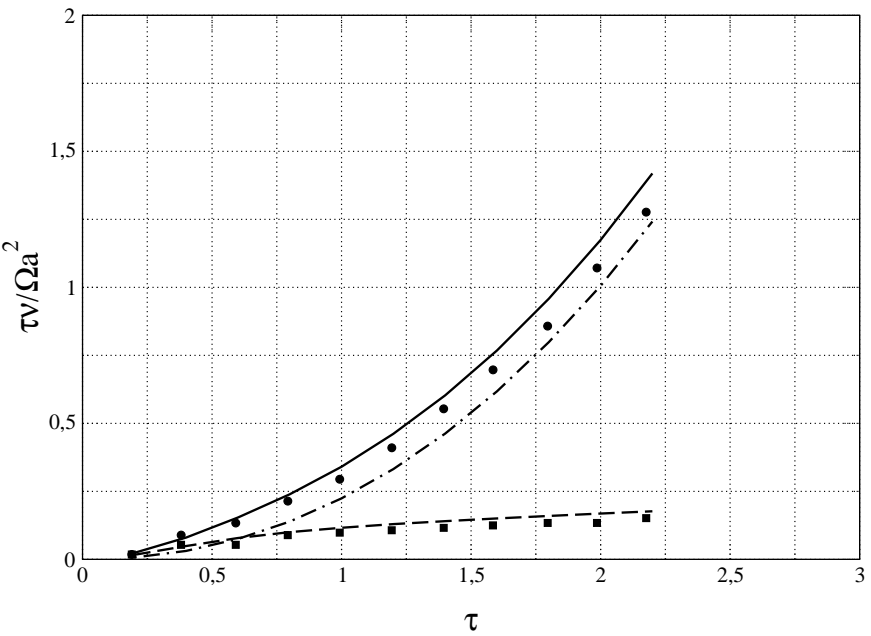

Fig. 9. Heigth-averaged normalized dynamic viscosity $\tau \bar{\nu}$ as a function of $\tau$. DSMC results: dashed line, kinetic contribution $\tau \overline{\nu^{\mathrm{k}}}$; dot-dashed line, collisional contribution $\tau \overline{\nu^{\mathrm{c}}}$; solid line, total $\tau \bar{\nu}$. MD results from WT: $\square, \tau \overline{\nu^{\mathrm{k}}}$; , total $\tau \bar{\nu}$

Finally, the DSMC findings have also been compared to the moment method solutions by Araki (1991), who solved Eq. (17) by forcing $f_{0}$ to be a triaxial Gaussian, as far as the velocity dependence is concerned. No a priori assumption was made about the density profile, which was computed from the vertical momentum balance. Araki's calculations suffered from numerical difficulties which prevented the obtaining of a solution for $\tau>0.7$, but gave good results where convergence was achieved.

As in the previous sub-section, the analysis starts from the characteristics of vertical density profiles.

The curves representing the dependence of central volume fraction on optical depth are shown in Fig. 10b.

DSMC results belong to two different data sets: in the first one the self-gravity parameter $G_{\mathrm{s}}$ was set equal to 7.4 to match Araki's conditions, whereas $G_{\mathrm{S}}=6.6$ was used to reproduce MD results in (Salo 1991a; 1991b), where a lower value for the particles bulk density was used.

The DSMC curve corresponding to $G_{\mathrm{s}}=7.4$ practically superimposes exactly onto Araki's data, thus confirming the good quality of the moment method results. As expected, simulating self-gravity by a $\tau$-independent vertical frequency enhancement leads to higher central volume fractions. In particular, Wisdom \& Tremaine's curve does not approach the values obtained with $G_{\mathrm{s}}=0$ at low $\tau$. On the contrary, Salo's results with the self-consistent evaluation of $F_{z}^{\mathrm{sg}}$ exhibits the correct behavior, and they are rather well reproduced by the DSMC simulations with $G_{\mathrm{s}}=6.6$.

The comparison with the case $G_{\mathrm{s}}=0$ (see Fig. 3) shows that the $\eta(0)-\tau$ curves displayed in Fig. 10 exhibit a more rapid growth, caused by the self-gravity field which is superimposed onto the gravitational field generated by the planet, thus enhancing ring flattening. Actually, at $\tau=0.2$ $F_{z}^{\mathrm{sg}}$ already exceeds the vertical component of the planet's gravity. The strong flattening can also be seen from the dispersion $\Delta z$ of the vertical density profile, reported in
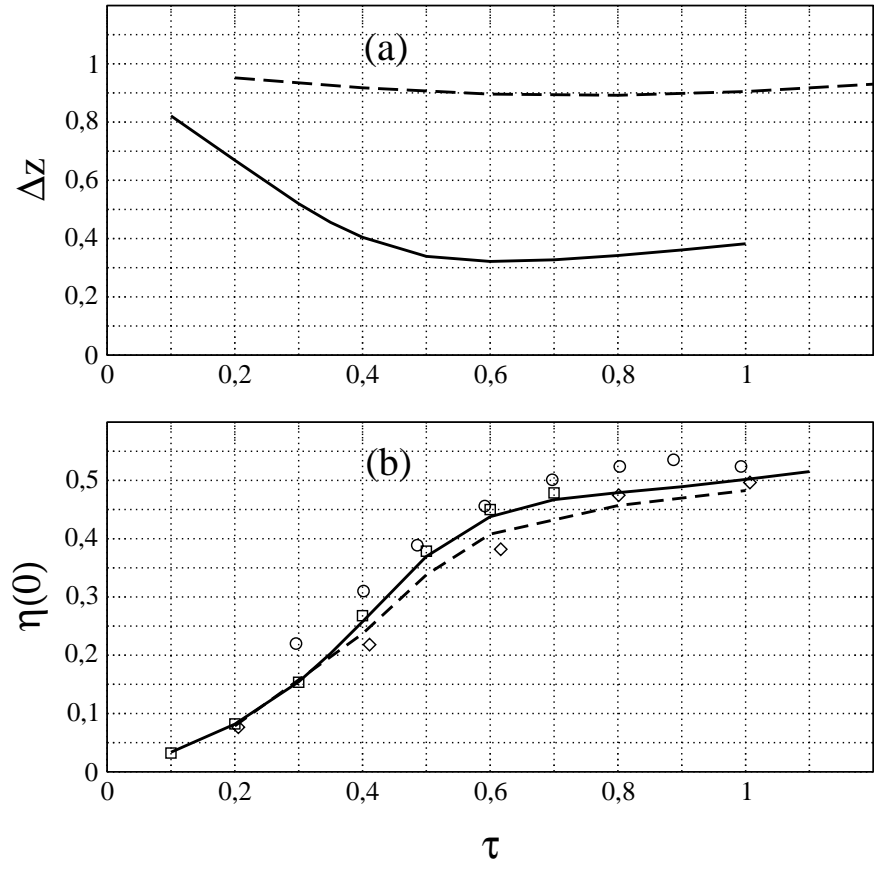

Fig. 10. Graph a): the standard deviation $\Delta z$ of particles displacements as a function of the optical depth $\tau$. Solid line, $e=0.5, \beta=-1, G_{\mathrm{s}}=7.4$; dashed line, $e=0.5, \beta=-1$, $G_{\mathrm{s}}=0$. Graph b): the volume fraction $\eta(0)$ in the center of the ring as a function of $\tau$. DSMC: solid line, $G_{\mathrm{s}}=7.4$; dashed line, $G_{\mathrm{s}}=6.6$. $\square$, moment method $G_{\mathrm{s}}=7.4$ (Araki 1991). MD simulations: $\circ$, WT's vertical frequency enhancement; $\diamond$, self-consistent gravity field (Salo 1991a, 1991b).

Fig. 10a as a function of $\tau$. The initially decreasing $\Delta z$ reaches a minimum around $\tau=0.6$, after which it starts growing. The minimum can be associated with the onset of vertical ring stratification (WT; Salo 1991a; 1991b), as shown in Fig. 11, which presents the evolution of the shape of the density profiles as $\tau$ ranges from 0.4 to 1.1.

Unfortunately, MD data for an accurate numerical comparison of density profiles can be easily extracted only from WT in the case $\tau=0.9$. Although the MD density profile has been obtained by the enhanced vertical frequency method, the comparison with DSMC is more than qualitative, since in this case the effective vertical frequency enhancement deduced from the derivative $\mathrm{d} F_{z}^{\mathrm{sg}} / \mathrm{d} z$ in $z=0$ turns out to be 3.55 . The DSMC curve correctly reproduces the position of the density peaks and the decreasing part of the MD density profile, however the density difference between the central minimum and the adjacent maxima is less pronounced, hence no local maximum of $\eta(0)$ is evident in the DSMC solutions. The deviation from MD data becomes stronger as $\tau$ increases and the volume fraction $\eta$ takes values well outside the validity limit of the Carnahan-Starling approximation. As is observed in other problems (Frezzotti 1997), although capable of correctly capturing the onset of stratification, the multilayered ring structure observed in MD simulations at high optical depth is out of reach of Enskog's Ansatz expressed by Eq. (11). The average velocity dispersions along the 


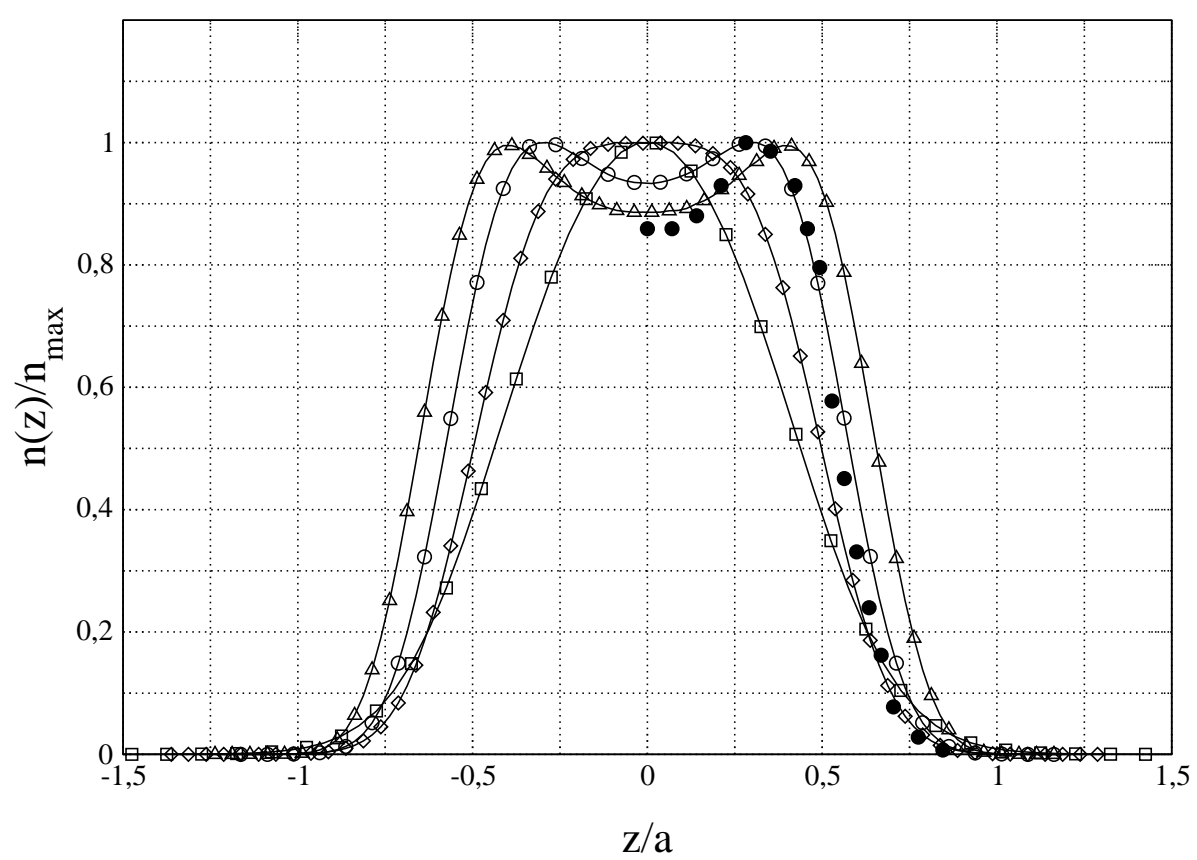

Fig. 11. Normalized density profiles. DSMC results, $e=0.5, \beta=-1, G_{\mathrm{s}}=7.4: \square, \tau=0.5 ; \diamond, \tau=0.7 ; \circ, \tau=0.9 ; \triangle, \tau=1.1 . \bullet$, WT's MD simulation with vertical frequency enhancement, $\tau=0.9$.

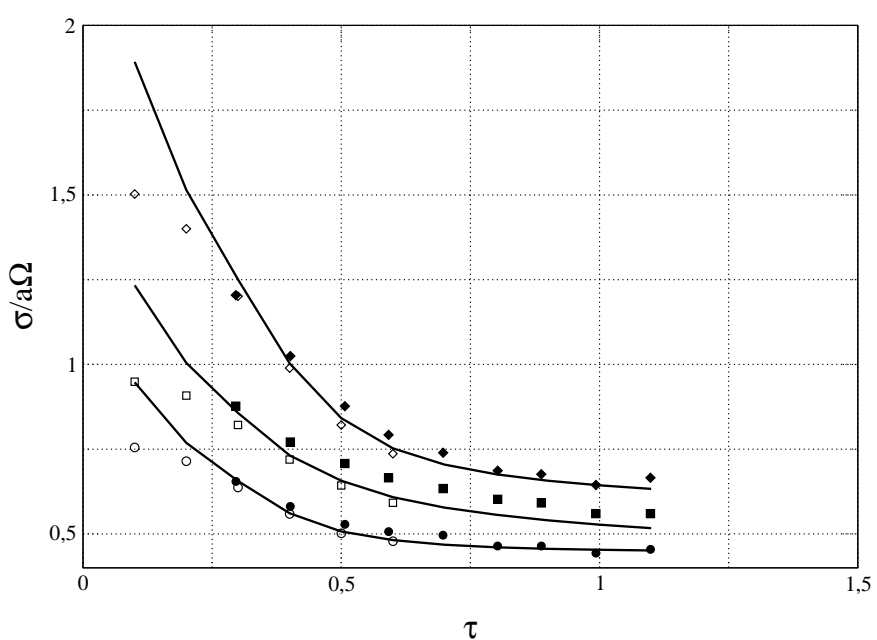

Fig. 12. Heigth-averaged velocity dispersions along the principal axes of the velocity ellipsoid. Values normalized to $a \Omega$. Solid curves represent DSMC results $\left(G_{\mathrm{s}}=7.4\right)$. Filled symbols MD simulations from WT: ○, $\overline{\sigma_{x}} ; \square, \overline{\sigma_{y}} ; \diamond, \overline{\sigma_{z}}$. White symbols represent results from moment method $\left(G_{\mathrm{s}}=7.4\right)$, (Araki 1991).

principal axes of the velocity ellipsoid are given in Fig. 12 . The DSMC results refer to the case $G_{\mathrm{S}}=7.4$ and they are compared with Araki's moment method solutions and WT molecular dynamics simulations which are extremely close to Salo's MD simulations with self-consistent treatment of $F_{z}^{\mathrm{sg}}$. Both DSMC and moment method solution of Eq. (17) are in good agreement with MD simulations, however DSMC deviates from Araki's predictions at low $\tau$ and gives results closer to the case $G_{\mathrm{S}}=0$. The analysis of the vertical thermal balance in the self-gravitating ring shows an interesting phenomenon which does not seem to have been noticed before. Plots of the relevant quantities in a low $\tau$ situation are given in Fig. 13.

A comparison with Figs. 7, 8 shows that in this case the qualitative behavior of the various quantities is the same as that exhibited when self-gravity is absent. In particular, the temperature plot has a single stationary point, a minimum, in the center of the ring, where the energy dissipation rate is higher than the energy production rate. However, when $\tau$ is set equal to 0.4 the temperature profile exhibits a completely different behavior with three stationary points: a maximum in the center and two symmetric minima. A look at the quantities appearing in the thermal balance equation, satisfied with very high accuracy, shows that the energy production rate in the ring center is now above the energy dissipation rate (see Fig. 14a) and that the heat flow has three zeroes. The first one is obviously in $z=0$ as before, the other two do not coincide with the temperature stationary points, but they are displaced slightly outward with respect to them.

If a hydrodynamic point of view is adopted, then the displacement indicates that a residual heat flux exists in the absence of a temperature gradient. In the case of systems composed of particles undergoing inelastic collisions, theory admits that in the hydrodynamic approximation heat flux can be written as:

$\boldsymbol{q}=-\kappa \nabla T-\mu \nabla n$

thus allowing for a contribution from the density gradient (Soto et al. 1999 and references therein). Values of the non-dimensional transport coefficient computed from MD simulations

$\hat{\mu}=\frac{n \mu}{T^{3 / 2}}$ 

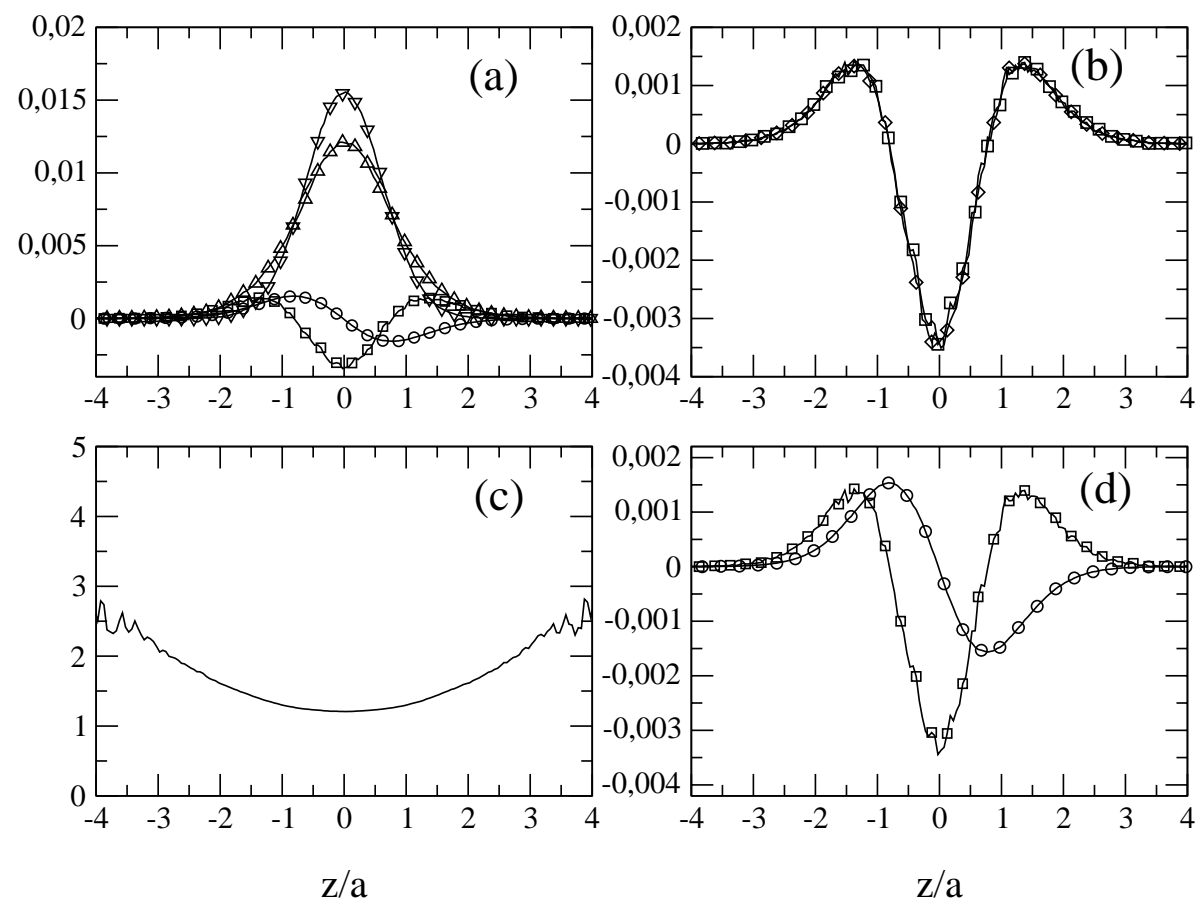

Fig. 13. Vertical thermal balance, $e=0.5, \beta=-1, G_{\mathrm{s}}=7.4, \tau=0.1$. Labeling of graphs and curves is the same as in Fig. 7 .
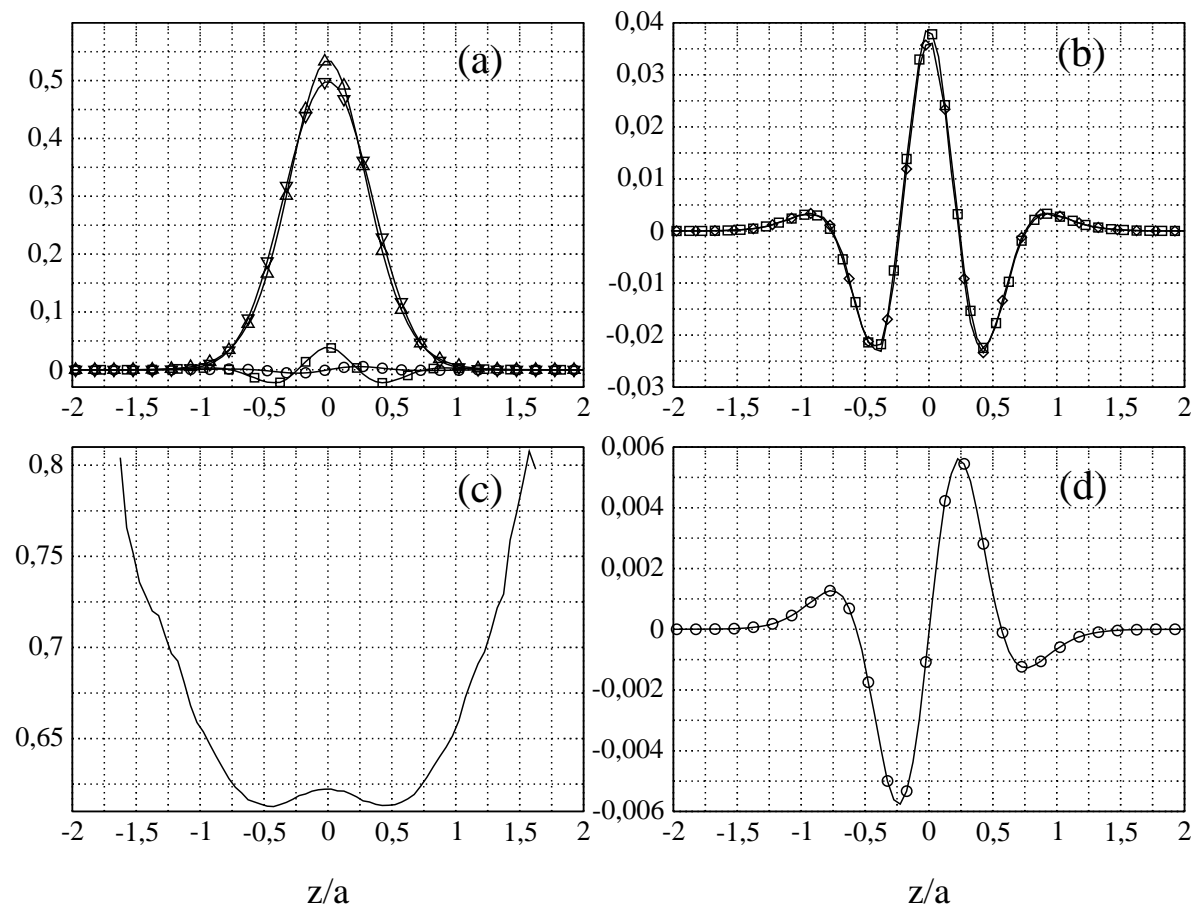

Fig. 14. Vertical thermal balance, $e=0.5, \beta=-1, G_{\mathrm{s}}=7.4, \tau=0.4$. Labeling of graphs and curves is the same as in Fig. 7 .

are given in Soto et al. (1999) as a function of density and normal restitution $e$ for small departures from the elastic case. The value of $\hat{\mu}$ that one can derive from the DSMC results in Fig. 14 is much smaller than the one that could be figured out by a reasonable extrapolation of the MD results. This discrepancy poses an interesting question which requires further investigation.

If the optical depth is further increased, the temperature minima move toward the low density region, where there are too few particles to obtain a good statistics. Therefore, Fig. 15c shows a temperature profile for $\tau=1.0$, which has a single maximum in $z=0$. The heat flow vector $\boldsymbol{q}(z)$ now points away from the ring center where viscous heating rate exceeds energy dissipation. The transition between the two kinds of solutions presented in Figs. $13-15$ occurs around $\tau=0.335$ if the self-gravity parameter $G_{\mathrm{s}}$ is equal to 7.4. At this particular optical depth, the viscous heating term, $\alpha P_{x y}$, reaches the intensity of 

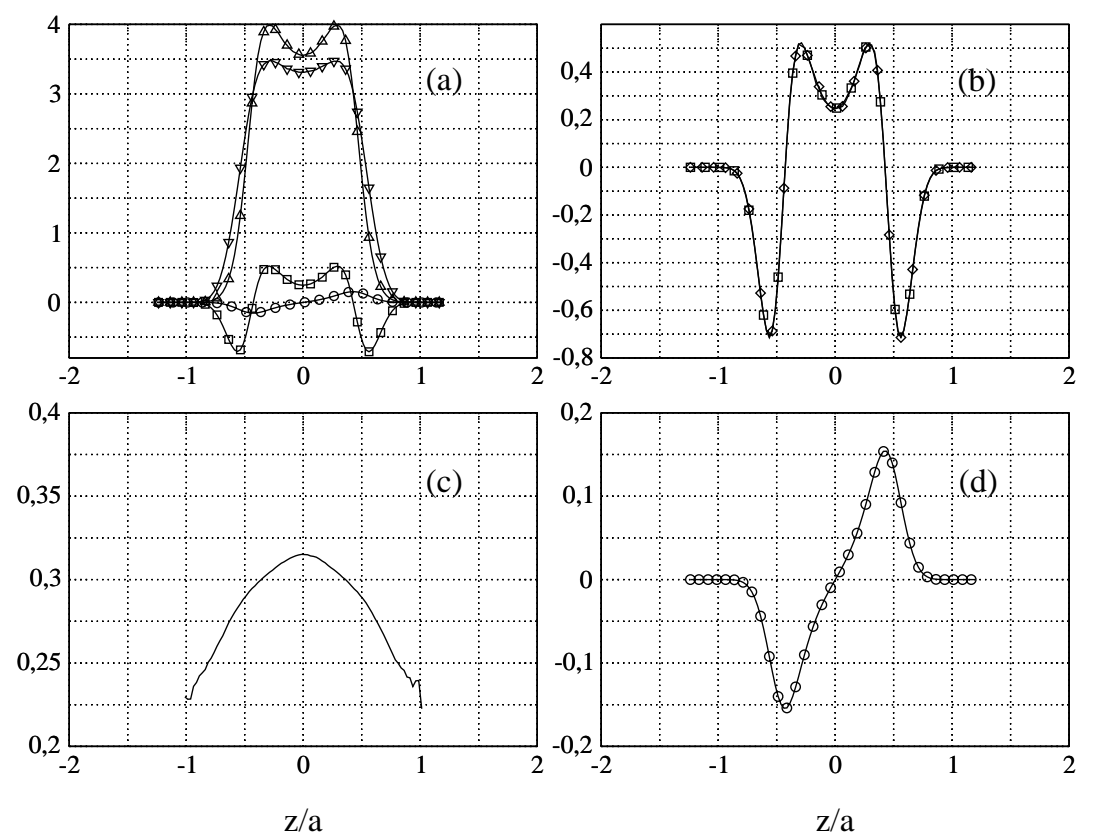

Fig. 15. Vertical thermal balance, $e=0.5, \beta=-1, G_{\mathrm{s}}=7.4, \tau=1.0$. Labeling of graphs and curves is the same as in Fig. 7 .

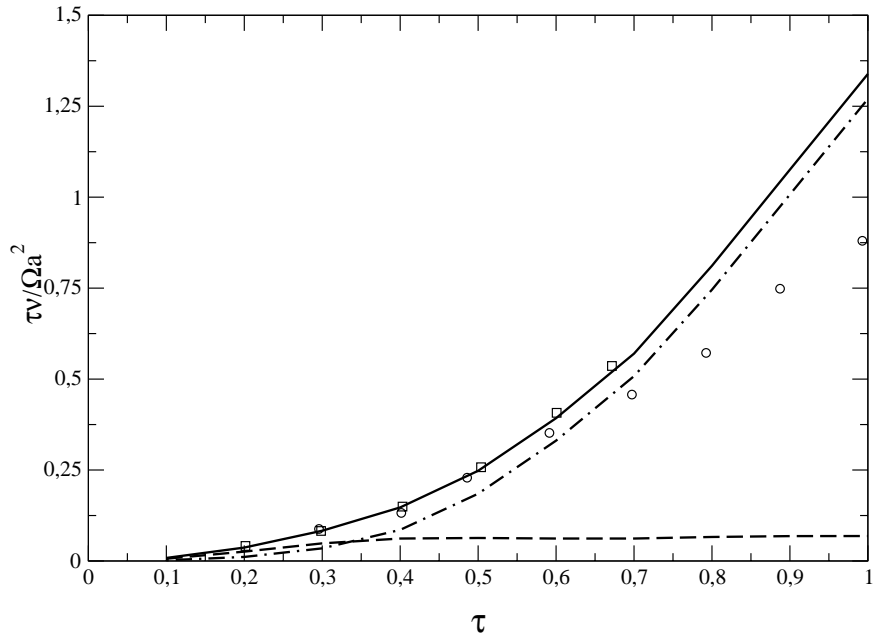

Fig. 16. Heigth-averaged normalized dynamic viscosity $\tau \bar{\nu}$ as a function of $\tau$. DSMC results: dashed line, kinetic contribution $\tau \overline{\nu^{\mathrm{k}}}$; dot-dashed line, collisional contribution $\tau \overline{\nu^{\mathrm{c}}}$; solid line, total $\tau \bar{\nu}$. MD results from WT: $\circ$, total $\tau \bar{\nu}$. Moment method values from (Araki 1991): $\square$, total $\tau \bar{\nu}$.

the rate of energy dissipation $D$. The explanation is not easy, but the transition seems to be due to ring narrowing induced by self-gravity. Actually the crossing of the $\alpha P_{x y}$ and $D$ curves cannot be attributed to a particular value of the ring density, since even higher density values are found when $G_{\mathrm{s}}$ is zero, but no inversion of the temperature profile is observed. The transition is not even related to ring stratification since it occurs well before $\tau \approx 0.7$, when the twin density peaks appear. It is worth mentioning that the quantities appearing in Eq. (47) have been computed by two completely different methods which yielded the same result. The first and simpler method obtains the dissipation rate as well as the stress tensor and heat flux components by accumulating energy and momentum exchanged in individual collisions at run time. The second one explicitly uses the definitions given by Eqs. (40), (43), (44), (45) and a Monte Carlo quadrature technique in which $f$ is sampled and discretized on a grid in the velocity space. Moreover, temperature profiles similar to those in Figs. 14, 15 seem to have been recently found in MD simulations (Salo 2000, private communication) in which the same constant value of the restitution coefficient has been used.

The direct comparison of DSMC with MD P and $\boldsymbol{q}$ profiles is again not possible, but it has to be limited to $\tau \bar{\nu}$ values shown in Fig. 16. The agreement with Araki's moment method calculations is excellent and the agreement with MD evaluations is surprisingly good up to an optical depth of about 0.7 . At higher $\tau$ kinetic theory predictions rapidly deviate from MD and at $\tau=1$ the difference between the two methods amounts to about $52 \%$ of the MD value. A few observations are now in order. First, the discrepancy cannot be attributed to the different treatment of the self-gravity field in the present and Wisdom \& Tremaine's work from which MD data are taken. Actually, the kinematic viscosity values given in (Salo 1991a, 1991b), where the same self-consistent evaluation of $F_{z}^{\mathrm{sg}}$ was used, are very close to those obtained by the vertical frequency enhancement method. Second, DSMC an MD predictions of $\tau \bar{\nu}$ start deviating at the onset of ring stratification, i.e. when the density profiles predicted by the two methods also show differences. Third and more important, when $\tau$ exceeds 0.7 the volume fraction $\eta$ turns out to be outside of the validity region of the Carnahan-Starling approximation in a more or less large portion of the ring thickness (see Figs. 10b, 11). As is well known, Eq. (12) provides an 

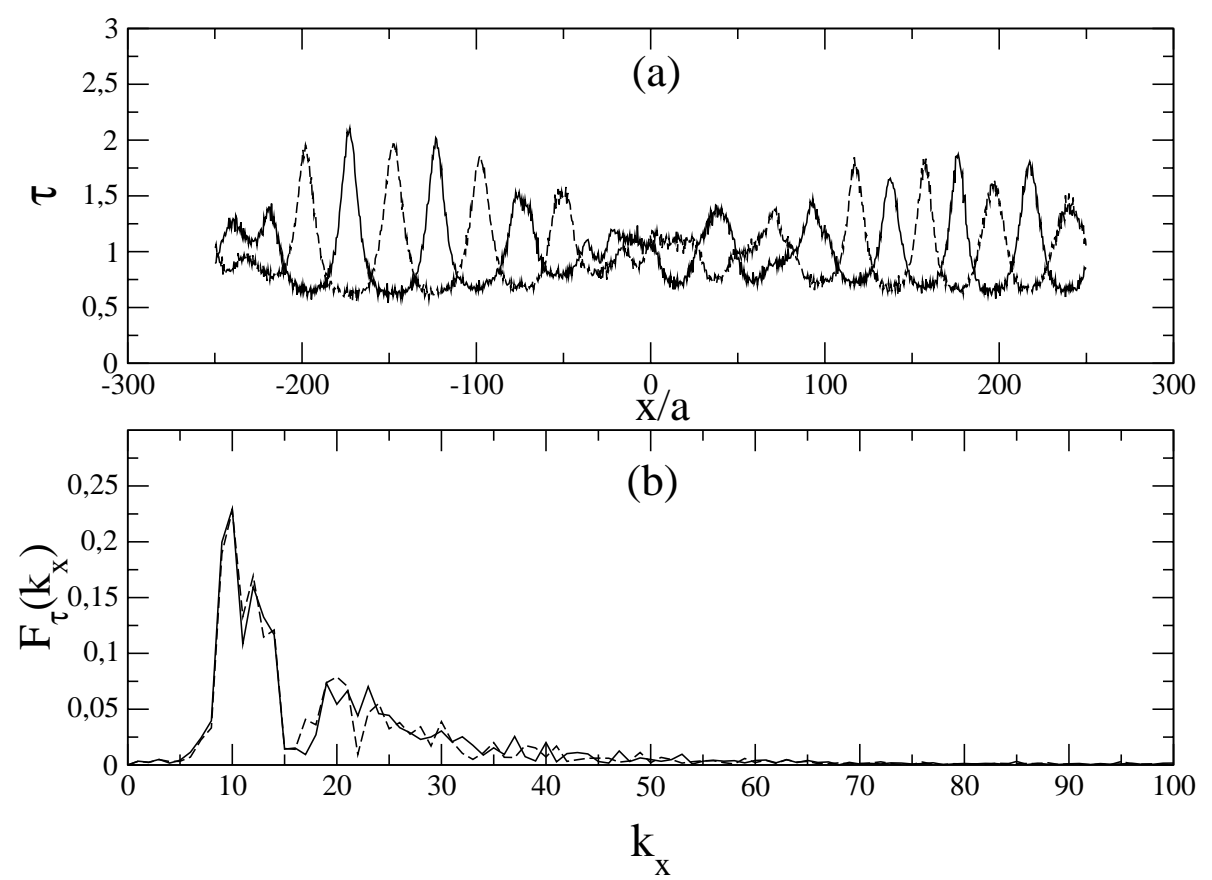

Fig. 17. Graph a): radial optical depth profiles after 97 (solid line) and 97.5 (dashed line) revolutions. Graph b): Fourier transforms of $\tau$ profiles showed in graph a); $k_{x}$ is the wave number.

extremely good approximation of the equation of state of the hard sphere fluid, but considerably overestimates pressure, $Y$ factor as well as collision rate beyond $\eta \approx 0.47$, where the hard sphere fluid exhibits a liquid-solid phase transition (Balescu 1975). For instance, at $\eta=0.56$, the peak value of the DSMC solution with $\tau=1.1$, Eq. (12) gives a $Y$ value which is about $60 \%$ higher than the correct one. It is clear that the above argument applies to a hard sphere fluid in uniform equilibrium. No phase transition occurs when the density has strong gradients and exceeds the 0.47 limit only locally. In this case the accuracy of Eq. (12) has not been systematically assessed, but the present results indicate that the kinetic theory results are in good agreement with MD only within the strict validity limits of the Carnahan-Starling approximation.

\section{Conclusions}

As stated in the introduction, the aim of the present work is the assessment of the merits and deficiencies of a theoretical model of dense granular fluids which is based on Enskog's kinetic equation and a simplified treatment of pair correlations. The resulting kinetic equation is solved directly by a statistical Monte Carlo method which does not need that further assumptions or approximations are made about the distribution function.

The results lead us to conclude that the present numerical method, although essentially confirming the findings of previous kinetic theory studies based on moment method approximations, gives a more detailed description of the ring vertical structure. In particular, the vertical profiles of the stress tensor and heat flow vector components have been computed and the peculiar behavior of the granular temperature in the self-gravitating case has been pointed out. In particular, two aspects, related to granular temperature profiles, seem to deserve further investigation. The first one is the quantitative comparison with the hydrodynamic prediction of the heat flux in general and, in particular, of its component proportional to the density gradient. The second one is related to the effects that velocity-dependent restitution coefficients might have on temperature profiles exhibiting a maximum in the ring center. The comparison with MD simulations shows that Enskog theory provides good results even at relatively high optical depths, if self-gravitation is ignored. Its deficiencies appear in dealing with strongly flattened rings when self-gravitation is switched on. As explained in Sect. 4, the simple approximation used to take pair correlations into account, although capable of predicting the correct critical $\tau$ for the onset of ring stratification, fails to reproduce MD density profiles at higher optical depths. Although stratification might be considered a secondary aspect related to the one-dimensional geometry of the problem, the discrepancy between MD and DSMC values of heigth-integrated dynamic viscosity observed at $\tau>0.7$ poses more serious limitations to the present approach, since it might prevent one from obtaining accurate results in two or three-dimensional simulations of oscillatory ring instabilities.

This seems to be confirmed by the results of a few preliminary three-dimensional DSMC simulations in which Eq. (14) is solved in a rectangular ring patch whose extent in the radial direction is $500 a$ and contains 400000 particles. The average optical depth is set equal to 1 and vertical self-gravity is simulated by a vertical frequency enhancement factor equal to 3.0. As Fig. 17 shows, the 
flowfield, initially uniform, spontaneously develops oscillating density waves. The typical wavelength observed in DSMC simulations (about 50a) turns out to be much shorter than 100-200a, as predicted by similar MD simulations (Salo et al. 2001).

It is clear that the limitations indicated by the present study would also be inherited by any hydrodynamic approximation based in Eqs. (14), (12) and that an improvement of the theory can only come from a better model for pair correlations which, in the spirit of the Revised Enskog Theory (RET) for the hard sphere fluid (van Beijren \& Ernst 1973), should take into account the effects of density gradients on pair correlation patterns.

Acknowledgements. The Author wishes to thank Dr. J. Schmidt and Prof. F. Spahn for many illuminating discussions and Prof. H. Salo for providing his numerical results.

\section{References}

Araki, S. 1988, Icarus, 76, 182

Araki, S. 1991, Icarus, 90, 139

Araki, S., \& Tremaine, S. 1986, Icarus, 65, 83

Balescu, R. 1975, Equilibrium and Nonequilibrium Statistical Mechanics (John Wiley \& Sons, New York)

Bird, G. A. 1994, Molecular Gas Dynamics and the Direct Simulation of Gas Flows (Clarendon Press, Oxford)

Borderies, N., Goldreich, P., \& Tremaine, S. 1985, Icarus, 63, 406

Brey, J. J., Ruiz-Montero, M. J., \& Cubero, D. 1996, Phys. Rev. E, 54, 3664

Brilliantov, N., \& Pöschel, T. 2001, Granular Gases with Impact-Velocity-Dependent Restitution Coefficient, in Lecture Notes in Physics, ed. T., Pöschel, S., Luding (Springer, Berlin) 564, 100

Carnahan, N. F., \& Starling, K. E. 1969, J. Chem. Phys., 51, 635

Cercignani, C. 2000, Rarefied Gas Dynamics (Cambridge University Press, Cambridge, UK)
Cercignani, C., \& Lampis, M. 1988, J. Stat. Phys., 53, 655

Enskog, D. 1922, Kungl. Svenska Vetenskapakad. Handl., 63, 3

Frezzotti, A. 1997, Phys. Fluids, 9, 1329

Frezzotti, A. 1999, Applications of Enskog theory to the study of granular fluids, in Proc. of the 21st International Symp. on Rarefied Gas Dynamics, ed. R., Brun, R., Campargue (CEPADUES, Toulouse), 2, 93

Frezzotti, A. 2000, Physica A, 278, 161

Goldreich, P., \& Tremaine, S. 1978, Icarus, 34, 227

Goldshtein, A., \& Shapiro, M. 1995, J. Fluid Mech., 282, 75

Hill, G. W. 1878, Am. J. Math., 1, 5

Koura, K. 1986, Phys. Fluids A, 29, 3509

Lun, C. K. K., Savage, S. B. 1987, Trans. ASME E: J. Appl. Mech., 54, 47

Mosqueira, I. 1996, Icarus, 122, 128

Papaloizou, J. C. B., \& Lin, D. N. C. 1988, ApJ, 331, 838

Resibois, P., \& DeLeener, M. 1977, Classical kinetic theory of fluids (J. Wiley \& Sons, New York)

Salo, H., 1991, Icarus 90, 254

Salo, H. 1991, Icarus, 92, 367

Salo, H. 1995, Icarus, 117, 287

Salo, H., Schmidt, J., \& Spahn, F. 2001, Viscous Overstability in Saturn's B-Ring: I. Direct simulations and measurments of transport coefficients, Icarus, submitted

Schmidt, J., Salo, H., Petzschmann, O., \& Spahn, F. 1999, A\&A, 345, 646

Schmidt, J., Salo, H., Spahn, F., \& Petzschmann, O. 2001, Viscous Overstability in Saturn's B-Ring: II. Hydrodynamic theory and comparison to simulations, Icarus, submitted

Schmit, U., \& Tscharnuter, W. M. 1995, Icarus, 115, 304

Schmit, U., \& Tscharnuter, W. M. 1999, Icarus, 138, 173

Simon, V., \& Jenkins, J. T. 1994, Icarus, 110, 109

Soto, R., Mareschal, M., \& Risso, D. 1999, Phys. Rev. Lett., 83,5003

Spahn, F., Schmidt, J., \& Salo, H. 2000, Icarus, 145, 657

van Beijeren, H., \& Ernst, M. H. 1973, Physica, 68, 437

van Noije, T. P. C., Ernst, M. H., \& Brito, R. 1998, Physica A, 251, 266

Wisdom, J., Tremaine, S. 1988, Astron. J., 95, 925 